\title{
Approximation Schemes for Low-Rank Binary Matrix Approximation Problems*
}

\author{
Fedor V. Fomin ${ }^{\dagger} \quad$ Petr A. Golovach ${ }^{\dagger} \quad$ Daniel Lokshtanov $^{\dagger} \quad$ Fahad Panolan $^{\dagger}$ \\ Saket Saurabh ${ }^{\dagger}$
}

\begin{abstract}
We provide a randomized linear time approximation scheme for a generic problem about clustering of binary vectors subject to additional constrains. The new constrained clustering problem encompasses a number of problems and by solving it, we obtain the first linear timeapproximation schemes for a number of well-studied fundamental problems concerning clustering of binary vectors and low-rank approximation of binary matrices. Among the problems solvable by our approach are Low GF(2)-RAnk Approximation, Low Boolean-RANK Approximation, and various versions of Binary Clustering. For example, for Low GF(2)-Rank Approximation problem, where for an $m \times n$ binary matrix $A$ and integer $r>0$, we seek for a binary matrix $B$ of $\mathrm{GF}(2)$ rank at most $r$ such that $\ell_{0}$ norm of matrix $\mathbf{A}-\mathbf{B}$ is minimum, our algorithm, for any $\epsilon>0$ in time $f(r, \epsilon) \cdot n \cdot m$, where $f$ is some computable function, outputs a $(1+\epsilon)$-approximate solution with probability at least $\left(1-\frac{1}{e}\right)$. Our approximation algorithms substantially improve the running times and approximation factors of previous works. We also give (deterministic) PTASes for these problems running in time $n^{f(r) \frac{1}{\epsilon^{2}} \log \frac{1}{\epsilon}}$, where $f$ is some function depending on the problem. Our algorithm for the constrained clustering problem is based on a novel sampling lemma, which is interesting in its own.
\end{abstract}

\footnotetext{
${ }^{*}$ The research leading to these results have been supported by the Research Council of Norway via the projects "CLASSIS" and "MULTIVAL".

${ }^{\dagger}$ Department of Informatics, University of Bergen, Norway.
} 


\section{Introduction}

We define a new clustering problem which encompasses a number of well studied problems about low-rank approximation of binary matrices and clustering of binary vectors.

In order to obtain approximation algorithms for low-rank approximation problem, we design approximation algorithms for a "constrained" version of binary clustering.

A $k$-ary relation $R$ is a set of binary $k$-tuples with elements from $\{0,1\}$. A $k$-tuple $t=$ $\left(t_{1}, \ldots, t_{k}\right)$ satisfies $R$, we write $t \in R$, if $t$ is equal to one of $k$-tuples from $R$.

Definition 1 (Vectors satisfying $\mathcal{R}$ ). Let $\mathcal{R}=\left\{R_{1}, \ldots, R_{m}\right\}$ be a set of $k$-ary relations. We say that a set $C=\left\{\mathbf{c}_{1}, \mathbf{c}_{2}, \ldots, \mathbf{c}_{k}\right\}$ of binary $m$-dimensional vectors satisfies $\mathcal{R}$ and write $\langle C, \mathcal{R}\rangle$, if $\left(\mathbf{c}_{1}[i], \ldots, \mathbf{c}_{k}[i]\right) \in R_{i}$ for all $i \in\{1, \ldots, m\}$.

For example, for $m=2, k=3, R_{1}=\{(0,0,1),(1,0,0)\}$, and $R_{2}=\{(1,1,1),(1,0,1),(0,0,1)\}$, the set of vectors

$$
\mathbf{c}_{1}=\left(\begin{array}{l}
0 \\
1
\end{array}\right), \mathbf{c}_{2}=\left(\begin{array}{l}
0 \\
0
\end{array}\right), \mathbf{c}_{3}=\left(\begin{array}{l}
1 \\
1
\end{array}\right)
$$

satisfies $\mathcal{R}=\left\{R_{1}, R_{2}\right\}$ because $\left(\mathbf{c}_{1}[1], \mathbf{c}_{2}[1], \mathbf{c}_{3}[1]\right)=(0,0,1) \in R_{1}$ and $\left(\mathbf{c}_{1}[2], \mathbf{c}_{2}[2], \mathbf{c}_{3}[2]\right)=$ $(1,0,1) \in R_{2}$.

Let us recall that the Hamming distance between two vectors $\mathbf{x}, \mathbf{y} \in\{0,1\}^{m}$, where $\mathbf{x}=$ $\left(x_{1}, \ldots, x_{m}\right)^{\top}$ and $\mathbf{y}=\left(y_{1}, \ldots, y_{m}\right)^{\top}$, is $d_{H}(\mathbf{x}, \mathbf{y})=\sum_{i=1}^{m}\left|x_{i}-y_{i}\right|$ or, in words, the number of positions $i \in\{1, \ldots, m\}$ where $x_{i}$ and $y_{i}$ differ. For a set of vectors $C$ and vector $x$, we define $d_{H}(\mathbf{x}, C)$, the Hamming distance between $x$ and $C$, as the minimum Hamming distance between $x$ and a vector from $C$. Thus $d_{H}(\mathbf{x}, C)=\min _{\mathbf{c} \in C} d_{H}(\mathbf{x}, \mathbf{c})$.

Then we define the following problem.

Binary Constrained Clustering

Input: A set $X \subseteq\{0,1\}^{m}$ of $n$ vectors, a positive integer $k$ and a set of $k$-ary relations $\mathcal{R}=\left\{R_{1}, \ldots, R_{m}\right\}$.

Task: Among all vector sets $C=\left\{\mathbf{c}_{1}, \ldots, \mathbf{c}_{k}\right\} \subseteq\{0,1\}^{m}$ satisfying $\mathcal{R}$, find a set $C$ minimizing the sum $\sum_{\mathbf{x} \in X} d_{H}(\mathbf{x}, C)$.

First we prove the following theorem.

Theorem 1. There is a deterministic algorithm which given instance of BINARY CONSTRAINED CLUSTERING and $\epsilon>0$, runs in time $m \cdot n^{\mathcal{O}\left(\frac{k^{2}}{\epsilon^{2}} \log \frac{1}{\epsilon}\right)}$ and outputs a $(1+\epsilon)$-approximate solution.

Our main result is the following theorem.

Theorem 2. There is an algorithm which for a given instance of BINARY CONSTRAINED ClusTERING and $\epsilon>0$ in time $2^{\mathcal{O}\left(\frac{k^{4}}{\varepsilon^{2}} \log \frac{1}{\varepsilon}\right)} \cdot\left(\frac{1}{\varepsilon}\right)^{\mathcal{O}\left(\frac{k}{\varepsilon^{2}} \log \frac{1}{\varepsilon}\right)} n \cdot m$ outputs a $(1+\epsilon)$-approximate solution with probability at least $\left(1-\frac{1}{e}\right)$.

In other words, the algorithm outputs a set of vectors $C=\left\{\mathbf{c}_{1}, \ldots, \mathbf{c}_{k}\right\} \subseteq\{0,1\}^{m}$ satisfying $\mathcal{R}$ such that $\sum_{\mathbf{x} \in X} d_{H}(\mathbf{x}, C) \leq(1+\epsilon) \cdot O P T$, where $O P T$ is the value of the optimal solution.

Theorems 1 and 2 have a number of interesting applications. 


\subsection{Applications of the main theorem}

Binary matrix factorization is the following problem. Given a binary $m \times n$ matrix, that is a matrix with entries from domain $\{0,1\}$,

$$
\mathbf{A}=\left(\begin{array}{cccc}
a_{11} & a_{12} & \ldots & a_{1 n} \\
a_{21} & a_{21} & \ldots & a_{2 n} \\
\vdots & \vdots & \ddots & \vdots \\
a_{m 1} & a_{m 2} & \ldots & a_{m n}
\end{array}\right)=\left(a_{i j}\right) \in\{0,1\}^{m \times n}
$$

the task is to find a "simple" binary $m \times n$ matrix B which approximates A subject to some specified constrains. One of the most widely studied error measures is the Frobenius norm, which for the matrix $\mathbf{A}$ is defined as

$$
\|\mathbf{A}\|_{F}=\sqrt{\sum_{i=1}^{m} \sum_{j=1}^{n}\left|a_{i j}\right|^{2}} .
$$

Here the sums are taken over $\mathbb{R}$. Then we want to find a matrix $\mathbf{B}$ with certain properties such that

$$
\|\mathbf{A}-\mathbf{B}\|_{F}^{2}
$$

is minimum.

In particular, two variants of the problem were studied in the literature, in the first variant on seeks for a matrix $\mathbf{B}$ of small GF(2)-rank. In the second variant, matrix $\mathbf{B}$ should be of Boolean rank $r$. Depending on the selection of the rank, we obtain two different optimization problems.

Low GF(2)-Rank Approximation. Here the task is to approximate a given binary matrix A by $\mathbf{B}$ that has $\mathrm{GF}(2)$-rank $r$.

Low GF(2)-Rank Approximation

Input: An $m \times n$-matrix $\mathbf{A}$ over $\mathrm{GF}(2)$ and a positive integer $r$.

Task: Find a binary $m \times n$-matrix $\mathbf{B}$ with $G F(2)$-rank $(\mathbf{B}) \leq r$ such that $\|\mathbf{A}-\mathbf{B}\|_{F}^{2}$ is minimum.

Low Boolean-Rank Approximation. Let $\mathbf{A}$ be a binary $m \times n$ matrix. Now we consider the elements of $\mathbf{A}$ to be Boolean variables. The Boolean rank of $\mathbf{A}$ is the minimum $r$ such that $\mathbf{A}=\mathbf{U} \wedge \mathbf{V}$ for a Boolean $m \times r$ matrix $\mathbf{U}$ and a Boolean $r \times n$ matrix $\mathbf{V}$, where the product is Boolean, that is, the logical $\wedge$ plays the role of multiplication and $\vee$ the role of sum. Here $0 \wedge 0=0,0 \wedge 1=0,1 \wedge 1=1,0 \vee 0=0,0 \vee 1=1$, and $1 \vee 1=1$. Thus the matrix product is over the Boolean semi-ring $(0,1, \wedge, \vee)$. This can be equivalently expressed as the normal matrix product with addition defined as $1+1=1$. Binary matrices equipped with such algebra are called Boolean matrices.

\section{Low Boolean-Rank Approximation}

Input: A Boolean $m \times n$ matrix $\mathbf{A}$ and a positive integer $r$.

Task: Find a Boolean $m \times n$ matrix $\mathbf{B}$ of Boolean rank at most $r$ such that $\|\mathbf{A}-\mathbf{B}\|_{F}^{2}$ is minimum.

Low-rank matrix approximation problems can be also treated as special cases of BINARY Constrained Clustering. 
Lemma 1. For any instance (A, $r$ ) of Low GF(2)-RANK Approximation, one can construct in time $\mathcal{O}\left(m+n+2^{2 r}\right)$ an instance $\left(X, k=2^{r}, \mathcal{R}\right)$ of Binary Constrained Clustering with the following property. Given any $\alpha$-approximate solution $C$ of $(X, k, \mathcal{R})$, an $\alpha$-approximate solution $\mathbf{B}$ of $(\mathbf{A}, r)$ can be constructed in time $\mathcal{O}(r m n)$ and vice versa.

Proof Sketch. Observe that if $\mathrm{GF}(2)-\operatorname{rank}(\mathbf{B}) \leq r$, then $\mathbf{B}$ has at most $2^{r}$ pairwise distinct columns, because each column is a linear combination of at most $r$ vectors of a basis of the column space of B. Also the task of Low GF(2)-RANK Approximation can equivalently be stated as follows: find vectors $\mathbf{s}_{1}, \ldots, \mathbf{s}_{r} \in\{0,1\}^{m}$ over $\mathrm{GF}(2)$ such that

$$
\sum_{i=1}^{n} \min \left\{d_{H}\left(\mathbf{s}, \mathbf{a}_{i}\right) \mid \mathbf{s} \text { is a linear combination of } \mathbf{s}_{1}, \ldots, \mathbf{s}_{r}\right\}
$$

is minimum, where $\mathbf{a}_{1}, \ldots, \mathbf{a}_{n}$ are the columns of $\mathbf{A}$. Respectively, to encode an instance of Low GF(2)-Rank Approximation as an instance of Binary Constrained Clustering, we construct the following relation $R$. Set $k=2^{r}$. Let $\Lambda=\left(\lambda_{1}, \ldots, \lambda_{k}\right)$ be the $k$-tuple composed by all pairwise distinct vectors of $\{0,1\}^{r}$. Thus each element $\lambda_{i} \in \Lambda$ is a binary $r$-vector. We define $R=\left\{\left(x^{\top} \lambda_{1}, \ldots, x^{\top} \lambda_{k}\right) \mid x \in\{0,1\}^{r}\right\}$. Thus $R$ consists of $2^{r} k$-tuples and every $k$-tuple in $R$ is a row of the matrix $\Lambda^{\top} \cdot \Lambda$. Now we define $X$ to be the set of columns of $\mathbf{A}$ and for each $i \in[m], R_{i}=R$. Note that since all $R_{i}$ are equal, we can construct and keep just one copy of $R$.

One can show that if $B$ is a solution to $(\mathbf{A}, r)$, then all linear combinations $C$ of a basis of the column vectors of $\mathbf{B}$ is a solution to $(X, k, \mathcal{R})$ and the cost of $C$ is at most the cost of the solution $\mathbf{B}$ of $(\mathbf{A}, r)$. Similarly, if $C$ is a solution to $(X, k, \mathcal{R})$, then solution $\mathbf{B}$ to $(\mathbf{A}, r)$ is constructed from $C$ by taking the the $j$-th column of $\mathbf{B}$ be equal to the vector in $C$ which is closest to the $j$-th column vector of $\mathbf{A}$. Clearly the cost of $\mathbf{B}$ is at most the cost of $C$. It is easy to see that given $\mathbf{B}$, one can construct $C$ in $\mathcal{O}(r m n)$ time and vice versa.

Similarly for Low Boolean-Rank Approximation we have the following lemma.

Lemma 2. For any instance $(\mathbf{A}, r)$ of Low Boolean-Rank Approximation, one can construct in time $\mathcal{O}\left(m+n+2^{2 r}\right)$ an instance $\left(X, k=2^{r}, \mathcal{R}\right)$ of Binary Constrained Clustering with the following property. Given any $\alpha$-approximate solution $C$ of $(X, k, \mathcal{R})$ an $\alpha$-approximate solution $\mathbf{B}$ of $(\mathbf{A}, r)$ can be constructed in time $\mathcal{O}(r m n)$ and vice versa.

The proof essentially repeats the proof of Lemma 1 . We are now working with the Boolean semi-ring $(0,1, \wedge, \vee)$ but still we can use exactly the same trick to reduce Low BoolEAN-RANK Approximation to Binary Constrained Clustering. The only difference is that GF(2) summations and products are replaced by $\vee$ and $\wedge$ respectively in the definition of the relation $R$. Thus every $k$-tuple in $R$ is a row of the matrix $\Lambda^{\top} \wedge \Lambda$.

Hence to design approximation schemes for Low Boolean-Rank Approximation and LOW GF(2)-RANK Approximation, it suffice to give an approximation scheme for Binary Constrained Clustering. The main technical contribution of the paper is the proof of the following theorem.

For $\alpha>1$, we say that an algorithm is an $\alpha$-approximation algorithm for the low-rank approximation problem if for a matrix $A$ and an integer $r$ it outputs a matrix $B$ satisfying the required constrains such that $\|\mathbf{A}-\mathbf{B}\|_{F}^{2} \leq \alpha \cdot\left\|\mathbf{A}-\mathbf{B}_{r}\right\|_{F}^{2}$, where $\mathbf{B}_{r}=\operatorname{argmin}_{\operatorname{rank}\left(\mathbf{B}_{r}\right)=r} \| \mathbf{A}-$ $\mathbf{B}_{r} \|_{F}^{2}$. By Theorems 1 and 2 and Lemmata 1 and 2, we obtain the following.

Corollary 1. (i) There is a deterministic algorithm which for a given instance of Low BOOLEANRAnK Approximation (Low GF(2)-RANK Approximation) and $\epsilon>0$ in time $m \cdot n^{\mathcal{O}\left(\frac{2^{2 r}}{\epsilon^{2}} \log \frac{1}{\epsilon}\right)}$ outputs a $(1+\epsilon)$-approximate solution. (ii) There is an algorithm which for a given instance 
of Low Boolean-Rank Approximation (Low GF(2)-Rank Approximation) and $\epsilon>0$

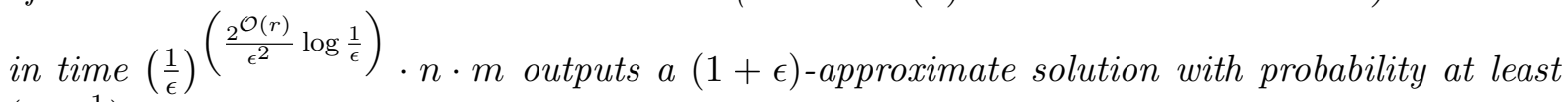
$\left(1-\frac{1}{e}\right)$.

Let us observe that our results also yield randomized approximation scheme for the "dual" maximization versions of the low-rank matrix approximation problems. In these problems one wants to maximize the number of elements that are the same in $A$ and $B$ or, in other words, to maximize the value of $n m-\|\mathbf{A}-\mathbf{B}\|_{F}^{2}$. It is easy to see that for every binary $m \times n$ matrix A there is a binary matrix $\mathbf{B}$ with $\operatorname{GF}(2)$-rank $(\mathbf{B}) \leq 1$ such that $\|\mathbf{A}-\mathbf{B}\|_{F}^{2} \leq m n / 2$. This observation implies that

$$
\begin{gathered}
\left(m n-\left\|\mathbf{A}-\mathbf{B}^{*}\right\|_{F}^{2}\right)-\left(m n-\|\mathbf{A}-\mathbf{B}\|_{F}^{2}\right)=\|\mathbf{A}-\mathbf{B}\|_{F}^{2}-\left\|\mathbf{A}-\mathbf{B}^{*}\right\|_{F}^{2} \leq \\
\varepsilon\left\|\mathbf{A}-\mathbf{B}^{*}\right\|_{F}^{2} \leq \varepsilon\left(m n-\left\|\mathbf{A}-\mathbf{B}^{*}\right\|_{F}^{2}\right) .
\end{gathered}
$$

\subsection{Binary clustering and variants}

The special case of Binary CONSTRAined Clustering where no constrains are imposed on the centers of the clusters is Binary $k$-MEAns.

BINARY $k$-MEANS

Input: A set $X \subseteq\{0,1\}^{m}$ of $n$ vectors and a positive integer $k$.

Task: Find a set $C=\left\{\mathbf{c}_{1}, \ldots, \mathbf{c}_{k}\right\} \subseteq\{0,1\}^{m}$ minimizing the sum $\sum_{\mathbf{x} \in X} d_{H}(\mathbf{x}, C)$.

Equivalently, in Binary $k$-MEANS we seek to partition a set of binary vectors $X$ into $k$ clusters $\left\{X_{1}, \ldots, X_{k}\right\}$ such that after we assign to each cluster its mean, which is a binary vector $c_{i}$ (not necessarily from $X$ ) closest to $X_{i}$, then the $\operatorname{sum} \sum_{i=1}^{k} \sum_{x \in X_{i}} d_{H}\left(\mathbf{c}_{i}, \mathbf{x}\right.$ ) is minimum.

Of course, Binary Constrained Clustering generalizes Binary $k$-Means: For given instance $(X, k)$ of BinaRY $k$-MEANs by taking sets $R_{i}, 1 \leq i \leq m$, consisting of all possible $k$ tuples $\{0,1\}^{k}$, we construct in time $\mathcal{O}\left(n+m+2^{k}\right)$ an instance $(X, k, \mathcal{R})$ of Binary Constrained Clustering equivalent to $(X, k)$. Note that since all the sets $R_{i}$ are the same, it is sufficient to keep just one copy of the set for the instance $(X, k, \mathcal{R})$. That is, any $(1+\varepsilon)$-approximation to one instance is also a $(1+\varepsilon)$-approximation to another. Theorems 1 and 2 implies that

Corollary 2. (i) There is a deterministic algorithm which for a given instance of BINARY $k$ MEANS and $\epsilon>0$ in time $m \cdot n^{\mathcal{O}\left(\frac{k^{2}}{\epsilon^{2}} \log \frac{1}{\epsilon}\right)}$ outputs a $(1+\epsilon)$-approximate solution. (ii) There is an algorithm which for a given instance of BINARY $k$-MEANS and $\epsilon>0$ in time $\left(\frac{1}{\epsilon}\right)^{\mathcal{O}\left(\frac{k^{4}}{\epsilon^{2}} \log \frac{1}{\epsilon}\right)} \cdot n \cdot m$ outputs a $(1+\epsilon)$-approximate solution with probability at least $\left(1-\frac{1}{e}\right)$.

Theorems 1 and 2 can be used for many other variants of binary clustering. Let us briefly mention some other clustering problems which fit in our framework.

For example, the following generalization of binary clustering can be formulated as BINARY Constrained Clustering. Here the centers of clusters are linear subspaces of bounded dimension $r$. (For $r=1$ this is Binary Constrained Clustering and for $c=1$ this is Low GF(2)-Rank Approximation.) More precisely, in Binary Projective Clustering we are given a set $X \subseteq\{0,1\}^{m}$ of $n$ vectors and positive integers $k$ and $r$. The task is to find a family of $r$-dimensional linear subspaces $C=\left\{C_{1}, \ldots, C_{k}\right\}$ over $\mathrm{GF}(2)$ minimizing the sum

$$
\sum_{\mathbf{x} \in X} d_{H}\left(\mathbf{x}, \cup_{i=1}^{k} C\right)
$$

To see that Binary Projective Clustering is the special case of Binary Constrained Clustering, we observe that the condition that $C_{i}$ is a $r$-dimensional subspace over $\operatorname{GF}(2)$ 
can be encoded (as in Lemma 1) by $2^{r}$ constrains. Similar arguments hold also for the variant of Binary Projective Clustering when instead of $r$-dimensional subspaces we use $r$-flats ( $r$-dimensional affine subspaces).

In Correlative $k$-Bicluster Editing, we are given a bipartite graph and the task is to change the minimum number of adjacencies such that the resulting graph is the disjoint union of at most $k$ complete bipartite graphs [3]. This is the special case of BinARY Constrained Clustering where each constrain $R_{i}$ consists of $k$-tuples and each of the $k$-tuples contains exactly one element 1 and all other elements 0 . Another problem which can be reduced to Binary Constrained Clustering is the following variant of the Biclustering problem [41]. Here for matrix $\mathbf{A}$, and positive integers $k, r$, we want to find a binary $m \times n$-matrix $\mathbf{B}$ such that $\mathbf{B}$ has at most $r$ pairwise-distinct rows, $k$ pairwise distinct-columns such that $\|\mathbf{A}-\mathbf{B}\|_{F}^{2}$ is minimum.

\subsection{Previous work}

Low-rank binary matrix approximation Low-rank matrix approximation is a fundamental and extremely well-studied problem. When the measure of the similarity between $\mathbf{A}$ and $\mathbf{B}$ is the Frobenius norm of matrix $\mathbf{A}-\mathbf{B}$, the rank- $r$ approximation (for any $r$ ) of matrix $\mathbf{A}$ can be efficiently found via the singular value decomposition (SVD). This is an extremely well-studied problem and we refer to surveys and books $[23,28,40]$ for an overview of this topic. However, SVD does not guarantee to find an optimal solution in the case when additional structural constrains on the low-rank approximation matrix B (like being non-negative or binary) are imposed. In fact, most of the variants of low-rank approximation with additional constraints are NP-hard.

For long time the predominant approaches for solving such low-rank approximation problems with NP-hard constrains were either heuristic methods based on convex relaxations or optimization methods. Recently, there has been considerable interest in the rigorous analysis of such problems $[4,11,33,36]$.

Low GF(2)-Rank Approximation arises naturally in applications involving binary data sets and serve as important tools in dimension reduction for high-dimensional data sets with binary attributes, see $[12,19,22,25,35,37,42]$ for further references. Due to the numerous applications of low-rank binary matrix approximation, various heuristic algorithms for these problems could be found in the literature [15, 21,22, 25, 37].

When it concerns a rigorous analysis of algorithms for Low GF(2)-RANK ApPRoximation, the previous results include the following. Gillis and Vavasis [16] and Dan et al. [12] have shown that Low GF(2)-RANK Approximation is NP-complete for every $r \geq 1$. A subset of the authors studied parameterized algorithms for Low GF(2)-RANK Approximation in [13].

The first approximation algorithm for Low GF(2)-RANK ApPROXIMATION is due to Shen et al. [37] who gave a 2-approximation algorithm for the special case of $r=1$. Shen et al. [37] formulated the rank-one problem as Integer Linear Programming and proved that its relaxation gives a 2-approximation. They also observed that the efficiency of their algorithm can be improved by reducing the linear program to the Max-Flow problem. Jiang et al. [22] found a much simpler algorithm by observing that for the rank-one case, simply selecting the best column of the input matrix yields a 2-approximation. Bringmann et al. [9] developed a 2approximation algorithm for $r=1$ which runs in sublinear time. Thus even for the special case $r=1$ no polynomial time approximation scheme was known prior to our work.

For rank $r>1$, Dan et al. [12] have shown that a $\left(r / 2+1+\frac{r}{2\left(2^{r}-1\right)}\right)$-approximate solution can be formed from $r$ columns of the input matrix A. Hence by trying all possible $r$ columns of A, we can obtain $r / 2+1+\frac{r}{2\left(2^{r}-1\right)}$-approximation in time $n^{\mathcal{O}(r)}$. Even the existence of a linear 
time algorithm with a constant-factor approximation for $r>1$ was open.

Low Boolean-Rank Approximation in case of $r=1$ coincides with Low GF(2)-RANK Approximation. Thus by the results of Gillis and Vavasis [16] and Dan et al. [12] Low Boolean-Rank Approximation is NP-complete already for $r=1$. While computing GF(2)rank (or rank over any other field) of a matrix can be performed in polynomial time, deciding whether the Boolean rank of a given matrix is at most $r$ is already an NP-complete problem. This follows from the well-known relation between the Boolean rank and covering edges of a bipartite graph by bicliques [18]. Thus for fixed $r$, the problem is solvable in time $2^{2^{\mathcal{O}(r)}}(n m)^{\mathcal{O}(1)}[14,17]$ and unless Exponential Time Hypothesis (ETH) fails, it cannot be solved in time $2^{2^{o(r)}}(n m)^{\mathcal{O}(1)}$ [10].

There is a large body of work on Low BooleAn-RANK Approximation, especially in the data mining and knowledge discovery communities. In data mining, matrix decompositions are often used to produce concise representations of data. Since much of the real data such as word-document data is binary or even Boolean in nature, Boolean low-rank approximation could provide a deeper insight into the semantics associated with the original matrix. There is a big body of work done on Low Boolean-Rank Approximation, see e.g. [7,8,12,27,29,30,38]. In the literature the problem appears under different names like Discrete Basis Problem [29] or Minimal Noise Role Mining Problem [27,31,39].

Since for $r=1$ Low Boolean-RAnK Approximation is equivalent to Low GF(2)-RANK Approximation, the 2-approximation algorithm for Low GF(2)-RAnK Approximation in the case of $r=1$ is also a 2-approximation algorithm for LOW BoOLEAN-RANK APPROXIMATiOn. For rank $r>1$, Dan et al. [12] described a procedure which produces a $2^{r-1}+1$ approximate solution to the problem.

Let us note that independently Ban et al. [6] obtained a very similar algorithmic result for low-rank binary approximation. Their algorithm runs in time $\left(\frac{1}{\epsilon}\right)^{2^{\mathcal{O}(r)} / \epsilon^{2}} n$. $m \cdot \log 2^{r} n$. Moreover, they also obtained a lower bound of $2^{2^{\delta r}}$ for a constant $\delta$ under Small Set Expansion Hypothesis and Exponential Time Hypothesis. Surprisingly, at first glance, the technique and approach in [6] to obtain algorithmic result for low-rank binary approximation is similar to that of ours.

Binary $k$-Means was introduced by Kleinberg, Papadimitriou, and Raghavan [24] as one of the examples of segmentation problems. Ostrovsky and Rabani [34] gave a randomized PTAS for BinaRY $k$-MEANS. In other words they show that for any $\gamma>0$ and $0<\varepsilon<1 / 8$ there is an algorithm finding an $(1+8 \varepsilon)^{2}$-approximate solution with probability at least $1-n^{-\gamma}$. The running time of the algorithm of Ostrovsky and Rabani is $n^{f(\varepsilon, k)}$ for some function $f$. No Efficient Polynomial Time Approximation Scheme (EPTAS), i.e. of running time $f(\varepsilon, k) \cdot n^{c}$, for this problem was known prior to our work.

For the dual maximization problem, where one wants to maximize $n m-\sum_{i=1}^{k} \sum_{x \in X_{i}} d_{H}\left(c_{i}, x\right)$ a significantly faster approximation is known. Alon and Sudakov [2] gave a randomized EPTAS. For a fixed $k$ and $\varepsilon>0$ the running time of the $(1-\varepsilon)$-approximation algorithm of Alon and Sudakov is linear in the input length.

BinaRY $k$-MEAns can be seen as a discrete variant of the well-known $k$-MEAns ClusterING. This problem has been studied thoroughly, particularly in the areas of computational geometry and machine learning. We refer to $[1,5,26]$ for further references to the works on $k$-Means Clustering. In particular, the ideas from the algorithm for $k$-Means ClusterING of Kumar et al. [26] form the starting point of our algorithm for BinARY Constrained Clustering. 


\subsection{Our approach.}

Sampling lemma and deterministic algorithm Our algorithms are based on Sampling Lemma (Lemma 3). Suppose we have a relation $R \subseteq\{0,1\}^{k}$ and weight tuple $\mathbf{w}=\left(w_{1}, \ldots, w_{k}\right)$, where $w_{i} \geq 0$ for all $i \in\{1, \ldots, k\}$. Then Sampling Lemma says that for any $\epsilon>0$, there is a constant $r=\Theta\left(\frac{k}{\varepsilon^{2}} \log \frac{1}{\varepsilon}\right)$ such that for any tuple $\mathbf{p}=\left(p_{1}, \ldots, p_{k}\right), 0 \leq p_{i} \leq 1, r$ random samples from Bernoulli distribution $B\left(p_{i}\right)$ for each $i \in\{1, \ldots, k\}$ gives a good estimate of the minimum weighted distance of $\mathbf{p}$ from the tuples in $R$. For more details we refer to Lemma 3.

Here we explain how our sampling lemma works to design a PTAS. Let $J=(X, k, \mathcal{R}=$ $\left.\left\{R_{1}, \ldots, R_{m}\right\}\right)$ of Binary Constrained Clustering, $\varepsilon>0$ be an instance Binary ConStrained Clustering and let $C=\left\{\mathbf{c}_{1}, \ldots, \mathbf{c}_{k}\right\}$ be an optimum solution to $J$. Let $X_{1} \uplus X_{2} \ldots \uplus$ $X_{k}$ be a partition of $X$ such that $\sum_{\mathbf{x} \in X} d_{H}(\mathbf{x}, C)=\sum_{i=1}^{k} \sum_{\mathbf{x} \in X_{i}} d_{H}\left(\mathbf{x}, \mathbf{c}_{i}\right)$. Informally, using Sampling Lemma, we prove that there is a constant $r=\Theta\left(\frac{k}{\varepsilon^{2}} \log \frac{1}{\varepsilon}\right)$ such that given $w_{i}=\left|X_{i}\right|$ and $r$ vectors uniformly at random chosen with repetition from $X_{i}$ for all $i \in\{1, \ldots, k\}$, we can compute in linear time a $(1+\epsilon)$-approximate solution to $J$ (see proof of Lemma 4 ). This immediately implies a PTAS for the problem.

Linear time algorithm (Theorem 2) The general idea of our algorithm for BINARY CONStrained Clustering is inspired by the algorithm of Kumar et al. [26]. Very informally, the algorithm of Kumar et al. [26] for $k$-Means Clustering is based on repeated sampling and does the following. For any (optimum) solution there is a cluster which size is at least $\frac{1}{k}$-th of the number of input vectors. Then when we sample a constant number of vectors from the input uniformly at random, with a good probability, the sampled vectors will be from the largest cluster. Moreover, if we sample sufficiently many (but still constant) number of vectors, they not only will belong to the largest cluster with a good probability, but taking the mean of the sample as the center of the whole cluster in the solution, we obtain a vector "close to optimum". This procedure succeeds if the size of the largest cluster is a large fraction of the number of vectors we used to sample from. Then the main idea behind the algorithm of Kumar et al. to assign vectors at a small distance from the guessed center vectors to their clusters. Moreover, once some vectors are assigned to clusters, then the next largest cluster will be a constant (depending on $k$ and $\epsilon$ ) fraction the size of the yet unassigned vectors. With the right choice of parameters, it is possible to show that with a good probability this procedure will be a good approximation to the optimum solution.

On a very superficial level we want to implement a similar procedure: iteratively sample, identify centers from samples, assign some of the unassigned vectors to the centers, then sample again, identify centers, etc. Unfortunately, it is not that simple. The main difficulty is that in Binary Constrained Clustering, even though we could guess vectors from the largest cluster, we cannot select a center vector for this cluster because the centers of "future" clusters should satisfy constrains from $\mathcal{R}$ - selection of one center could influence the "future" in a very bad way. Since we cannot select a good center, we cannot assign vectors to the cluster, and thus we cannot guarantee that sampling will find the next cluster. The whole procedure just falls apart!

Surprisingly, the sampling idea still works for Binary Constrained Clustering but we have to be more careful. The main idea behind our approach is that if we sample all "big" clusters simultaneously and assign the centers to these clusters such that the assigned centers "partially" satisfy $\mathcal{R}$, then with a good probability this choice does not mess up much the solution. After sampling vectors from all big clusters, we are left with two jobs- (i) find centers for the clusters sampled simultaneously and $(i i)$ these centers will be a subset of our final solution. The condition $(i)$ is guaranteed by our new Sampling Lemma (Lemma 5). Towards maintaining condition (ii), we prove that even after finding "approximately close centers" for 
the big clusters, there exist centers for the small clusters which together with the already found centers is a good approximate solution (i.e, they obey the relations and its cost is small). As far as we succeed in finding with a good probability a subset of "good" center vectors, we assign some of the remaining input vectors to the clusters around the centers. Then we can proceed iteratively.

Now we explain briefly how to obtain the running time to be linear. In each iteration after finding some center vectors we have mentioned that the vectors in the remaining input vectors which are close to already found centers can be safely assigned to the clusters of the center vectors already found. In fact we show that if the number of such vectors (vectors which are close to already found centers) are at most half the fraction of remaining input vectors, then there exist at least one cluster (whose center is yet be computed) which contains a constant fraction of the remaining set of vectors. In the other case we have that half fraction of the remaining vectors can be assigned to already found centers. This leads to a recurrence relation $T(n, k)=T\left(\frac{n}{2}, k\right)+c T\left(n, k-k^{\prime}\right)+c^{\prime} n \cdot m$ and $T(n, 0)=T(0, k)=1$, where $c$ and $c^{\prime}$ are constants depending on $k$ and $\epsilon$, and $k^{\prime} \geq 1$, provided we could find approximate cluster centers from the samples of large clusters in linear time. The above recurrence will solves to $f(k, \epsilon) n \cdot m$, for some function $f$. We need to make sure that we can compute cluster centers from the samples. In the case of designing a PTAS, we have already explained that we could compute approximate cluster centers using samples if know the size of each of those clusters. In fact we show that if the sizes of large clusters are comparable and know them approximately, then we could compute approximate cluster centers in linear time (see Lemma 5).

In Section 2, we give notations, definitions and some known results which we use through out the paper. In Section 3 we give notations related to Binary Constrained Clustering. In Section 4, we prove the important Sampling Lemma, which we use to design both PTAS and linear time randomized approximation scheme for Binary Constrained Clustering. Then in Section 5, we prove how Sampling Lemma can be used to get a (deterministic) PTAS for the problem. The subsequent sections are building towards obtaining a linear time approximation scheme for the problem.

\section{Preliminaries}

We use $\mathbb{N}$ to denote the set $\{1,2, \ldots\}$. For an integer $n \in \mathbb{N}$, we use $[n]$ as a shorthand for $\{1, \ldots, n\}$. For a set $U$ and non-negative integer $i, 2^{U}$ and $\left(\begin{array}{c}U \\ i\end{array}\right)$ denote the set of subsets of $U$ and set of $i$ sized subsets of $U$, respectively. For a tuple $b=\left(b_{1}, \ldots, b_{k}\right) \in\{0,1\}^{k}$ and an index $i \in\{1, \ldots, k\}$, we use $b[i]$ denotes the $i$ th entry of $t$, i.e, $b[i]=b_{i}$. We use log to denote the logarithm with base 2 .

In the course of our algorithm, we will be construction a solution iteratively. When we find a set of vectors $C=\left\{\mathbf{c}_{1}, \ldots, \mathbf{c}_{r}\right\}, r<k$, which will be a part of the solution, these vectors should satisfy relations $\mathcal{R}$. Thus we have to guarantee that for some index set $I \subset\{1, \ldots, k\}$ of size $r$, the set of vectors $C$ satisfies the part of $\mathcal{R}$ "projected" on $I$. More precisely,

Definition 2 (Projection of $\mathcal{R}$ on $I$, $\operatorname{proj}_{I}(\mathcal{R})$ ). Let $R \subseteq\{0,1\}^{k}$ be a relation and $I=$ $\left\{i_{1}, \ldots, i_{r}\right\} \subseteq\{1, \ldots, k\}$ be a subset of indices, where $i_{1}<i_{2}<\cdots<i_{r}$. We say that a relation $R^{\prime} \subseteq\{0,1\}^{r}$ is a projection of $R$ on $I$, denoted by $\operatorname{proj}_{I}(R)$, if $R^{\prime}$ is a set of $r$-tuples from $\{0,1\}^{r}$ such that $u=\left(u_{1}, \ldots, u_{r}\right) \in \operatorname{proj}_{I}(R)$ if and only if there exists $t \in R$ such that $t\left[i_{j}\right]=u_{j}$ for all $j \in\{1, \ldots, r\}$. In other words, the tuples of $\operatorname{proj}_{I}(R)$ are obtained from tuples of $R$ by leaving only the entries with coordinates from $I$. For a family $\mathcal{R}=\left\{R_{1}, \ldots, R_{m}\right\}$ of relations, where $R_{i} \subseteq\{0,1\}^{k}$, we use $\operatorname{proj}_{I}(\mathcal{R})$ to denote the family $\left\{\operatorname{proj}_{I}\left(R_{1}\right), \ldots, \operatorname{proj}_{I}\left(R_{m}\right)\right\}$.

Thus a set of vectors $\mathbf{c}_{1}, \ldots, \mathbf{c}_{r} \in\{0,1\}^{m}$ satisfies $\operatorname{proj}_{I}(\mathcal{R})$ if and only if for every $\ell \in$ $\{1, \ldots, m\}$ there exists $\mathbf{t} \in R_{\ell}$ such for every $j \in\{1, \ldots, r\}, \mathbf{c}_{\ell}[j]=\mathbf{t}\left[i_{j}\right]$. As far as we fix a 
part of the solution $C=\left\{\mathbf{c}_{1}, \ldots, \mathbf{c}_{r}\right\}$ and index set $I$ of size $r$, such that $C$ satisfies $\operatorname{proj}_{I}(\mathcal{R})$, we can reduce the family of relations $\mathcal{R}$ by deleting from each relation $R_{i} \in \mathcal{R}$ all $k$-tuples not compatible with $C$ and $I$. More precisely, for every $1 \leq i \leq m$, we can leave only $k$-tuples which projections on $I$ are equal to $\left\{\mathbf{c}_{1}[i], \ldots, \mathbf{c}_{r}[i]\right\}$. Let the reduced family of relations be $\left.\mathcal{R}\right|_{(I, C)}$. Then in every solution $S$ extending $C$, the set of vectors $S \backslash C$ should satisfy the projection of $\left.\mathcal{R}\right|_{(I, C)}$ on $\bar{I}=\{1, \ldots, k\} \backslash I$. This brings us to the following definitions.

Definition 3 (Reducing relations $\mathcal{R}$ to $\left.\left.\mathcal{R}\right|_{(I, C)}\right)$. Let $R \subseteq\{0,1\}^{k}$ be a relation and $I=$ $\left\{i_{1}, \ldots, i_{r}\right\} \subseteq\{1, \ldots, k\}$ be a subset of indices, where $i_{1}<i_{2}<\cdots<i_{r}$, and let $u=$ $\left(u_{1}, \ldots, u_{r}\right) \in \operatorname{proj}_{I}(R)$ be an $r$-tuple. We say that relation $R^{\prime} \subseteq R$ is obtained from $R$ subject to $I$ and $u$ and write $R^{\prime}=\left.R\right|_{(I, u)}$, if

$$
R^{\prime}=\left\{t \in R \mid t\left[i_{j}\right]=u_{j} \text { for all } j \in\{1, \ldots, r\}\right\} .
$$

For a set of vectors $C=\left\{\mathbf{c}_{1}, \ldots, \mathbf{c}_{r}\right\}$, set $I \subseteq\{1, \ldots, k\}$ of size $r$, and a family of relations $\mathcal{R}=\left\{R_{1}, \ldots, R_{m}\right\}$, we denote by $\left.\mathcal{R}\right|_{(I, C)}$ the family of relations $\left\{R_{1}^{\prime}, \ldots, R_{m}^{\prime}\right\}$, where $R_{i}^{\prime}=$ $\left.R_{i}\right|_{\left(I,\left(\mathbf{c}_{1}[i], \ldots, \mathbf{c}_{r}[i]\right)\right)}, 1 \leq i \leq m$.

Definition $4\left(\mathcal{R}(I, C)\right.$ : Projection of $\left.\mathcal{R}\right|_{(I, C)}$ on $\left.\bar{I}\right)$. For relation $R \subseteq\{0,1\}^{k}$, r-sized subset of indices $I \subseteq\{1, \ldots, k\}$ and $r$-tuple $u=\left(u_{1}, \ldots, u_{r}\right) \in \operatorname{proj}_{I}(R)$, we use $R(I, u)$ to denote the projection of $\left.R\right|_{(I, u)}$ on $\bar{I}=\{1, \ldots, k\} \backslash I$.

For a family $\mathcal{R}=\left\{R_{1}, \ldots, R_{m}\right\}$ of relations, set of $r \leq k$ vectors $C=\left\{\mathbf{c}_{1}, \ldots, \mathbf{c}_{r}\right\}$ from $\{0,1\}^{m}$, and r-sized set of indices $I \subseteq\{1, \ldots, k\}$, we use $\mathcal{R}(I, C)$ to denote the family $\left\{R_{1}^{\prime}, \ldots, R_{m}^{\prime}\right\}$, where $R_{i}^{\prime}=R_{i}\left(I, t_{i}\right)=\operatorname{proj}_{\bar{I}}\left(\left.R_{i}\right|_{\left(I, t_{i}\right)}\right)$ and $t_{i}=\left(\mathbf{c}_{1}[i], \ldots, \mathbf{c}_{r}[i]\right)$.

In other words, $R(I, u)$ consists of all $(k-r)$-tuples $v$, such that "merging" of $u$ and $v$ results in a $k$-tuple from $R$. In particular, the extension of $u$ and $I$ in $R$ can be generated by "merging" $u$ and all vectors of $R(I, u)$.

We also use $\mathbf{0}$ and $\mathbf{1}$ to denote vectors with all entries equal to 0 and 1 , respectively, where the dimension of the vectors will be clear from the context. For vector $\mathbf{x} \in\{0,1\}^{m}$ and set $X \subset\{0,1\}^{m}$, we use $d_{H}(\mathbf{x}, C)$ to denote the minimum Hamming distance (the number of different coordinates) between $\mathbf{x}$ and vectors in $C$. For sets $X, Y \subset\{0,1\}^{m}$, we define

$$
\operatorname{cost}(X, Y)=\sum_{\mathbf{x} \in X} d_{H}(\mathbf{x}, Y)
$$

For a vector $\mathbf{x} \in\{0,1\}^{m}$ and integer $\ell>0$, we use $\mathcal{B}(\mathbf{x}, \ell)$ to denote the open ball of radius $\ell$ centered in $x$, that is, the set of vectors in $\{0,1\}^{m}$ at Hamming distance less than $\ell$ from $\mathbf{x}$.

Probability. In the analysis of our algorithm we will be using well known tail inequalities like Markov's and Hoeffding's inequalities.

Proposition 3 (Markov's inequality [32]). Let $X$ be a non-negative random variable and $a>0$. Then

$$
\operatorname{Pr}(X \geq a \cdot \mathbf{E}[X]) \leq \frac{1}{a} .
$$

Proposition 4 (Hoeffding's inequality [20]). Let $X_{1}, \ldots, X_{n}$ be independent random variables such that each $X_{i}$ is strictly bounded by the intervals $\left[a_{i}, b_{i}\right]$. Let $X=\sum_{1}^{n} X_{i}$ and $t>0$. Then

$$
\operatorname{Pr}(X-\mathbf{E}[X] \geq t) \leq e^{\left(-\frac{2 t^{2}}{\left.\sum_{i \in[n]} b_{i}-a_{i}\right)^{2}}\right)} .
$$




\section{Notations related to Binary Constrained Clustering}

Let $J=\left(X, k, \mathcal{R}=\left\{R_{1}, \ldots, R_{m}\right\}\right)$ be an instance of Binary Constrained Clustering and $C=\left\{\mathbf{c}_{1}, \ldots, \mathbf{c}_{k}\right\}$ be a solution to $J$, that is, a set of vectors satisfying $\mathcal{R}$. Then the cost of $C$ is $\operatorname{cost}(X, C)$. Given set $C$, there is a natural way we can partition the set of vectors $X$ into $k$ sets $X_{1} \uplus \cdots \uplus X_{k}$ such that

$$
\operatorname{cost}(X, C)=\sum_{i=1}^{k} \operatorname{cost}\left(X_{i},\left\{\mathbf{c}_{i}\right\}\right)=\sum_{i=1}^{k} \sum_{\mathbf{x} \in X_{i}} d_{H}\left(\mathbf{x}, \mathbf{c}_{i}\right) .
$$

Thus for each vector $\mathbf{x}$ in $X_{i}$, the closest to $\mathbf{x}$ vector from $C$ is $\mathbf{c}_{i}$. We call such partition clustering of $X$ induced by $C$ and refer to sets $X_{1}, \ldots, X_{k}$ as to clusters corresponding to $C$.

We use OPT $(J)$ to denote the optimal solution to $J$. That is

$$
\mathrm{OPT}(J)=\min \{\operatorname{cost}(X, C) \mid<C, \mathcal{R}>\} .
$$

Note that in the definition of a vector set $C$ satisfiying relations $\mathcal{R}$, we require that the size of $C$ is $k$. We also need a relaxed notion for vector sets of size smaller than $k$ to satisfy a part of $\mathcal{R}$.

Definition 5 (Vectors respecting $\mathcal{R}$ ). Let $C=\left\{\mathbf{c}_{1}, \ldots, \mathbf{c}_{i}\right\} \subseteq\{0,1\}^{m}$ be a set of binary vectors, where $i \leq k$, we say that $C$ respects $\mathcal{R}$ if there is an index set $I \in\{1, \ldots, k\}$ such that $<$ $C, \operatorname{proj}_{I}(\mathcal{R})>$, that is, $C$ satisfies $\operatorname{proj}_{I}(\mathcal{R})$. In other words, $C$ is a solution to $\left(X, i, \operatorname{proj}_{I}(\mathcal{R})\right)$.

Notice that given a set $C^{\prime}$ of $i \leq k$ vectors which respects $\mathcal{R}$, one can extend it to a set $C$ in time linear in the size of $J$ such that $C$ satisfies $\mathcal{R}$. Thus $C$ is a (maybe non-optimal) solution to $J$ such that $\operatorname{cost}(X, C) \leq \operatorname{cost}\left(X, C^{\prime}\right)$. We will use this observation in several places and thus state it as a proposition.

Proposition 5. Let $J=\left(X, k, \mathcal{R}=\left\{R_{1}, \ldots, R_{m}\right\}\right)$ be an instance of BinaRY Constrained Clustering and $C^{\prime}=\left\{\mathbf{c}_{1}, \cdots, \mathbf{c}_{i}\right\} \subseteq\{0,1\}^{m}$ for $i \leq k$ be a set of vectors respecting $\mathcal{R}$. Then there is linear time algorithm which finds a solution $C$ to $J$ such that $\operatorname{cost}(X, C) \leq \operatorname{cost}\left(X, C^{\prime}\right)$.

Definition 6. Let $J=(X, k, \mathcal{R})$ be an instance of Binary Constrained Clustering. For $i \in\{1, \ldots, k\}$, we define

$$
\mathrm{OPT}_{i}(J)=\min \{\operatorname{cost}(X, C\}|| C \mid=i \text { and } C \text { respects } \mathcal{R}\} .
$$

An equivalent way of defining $\operatorname{OPT}_{i}(J)$ is

$$
\mathrm{OPT}_{i}(J)=\min \left\{\mathrm{OPT}\left(X, i, \operatorname{proj}_{I}(\mathcal{R})\right) \mid I \subseteq\{1, \ldots, k\} \text { and }|I|=i\right\} .
$$

Notice that

$$
\mathrm{OPT}_{1}(J) \geq \mathrm{OPT}_{2}(J) \geq \cdots \geq \mathrm{OPT}_{k}(J)=\mathrm{OPT}(J)
$$

\section{Sampling probability distributions}

One of the main ingredient of our algorithms is the lemma about sampling of specific probability distributions. To state the lemma we use the following notations. For a real $p$ between 0 and 1 we will denote by $B(p)$ the Bernoulli distribution which assigns probability $p$ to 1 and $1-p$ to 0 . We will write $X \sim B(p)$ to denote that $X$ is a random variable with distribution $B(p)$. 
Definition 7 (Weighted distance $\left.d^{\mathbf{w}}\right)$. For two $k$-tuples $\mathbf{u}=\left(u_{1}, \ldots, u_{k}\right)$ and $\mathbf{v}=\left(v_{1}, \ldots, v_{k}\right)$ over reals and $k$-tuple $\mathbf{w}=\left(w_{1}, \ldots, w_{k}\right)$ with $w_{i} \geq 0$, the distance from $\mathbf{u}$ to $\mathbf{v}$ weighted by $\mathbf{w}$ is defined as

$$
d^{\mathbf{w}}(\mathbf{u}, \mathbf{v})=\sum_{i=1}^{k} w_{i}\left|u_{i}-v_{i}\right| .
$$

Informally, Sampling Lemma proves the following. For an integer $k$, a relation $R \subseteq\{0,1\}^{k}$, a sequence of probability distribution $\mathbf{p}=\left(p_{1}, \ldots, p_{k}\right)$ and $\epsilon>0$, there is a constant $r$ (depending on $k$ and $\epsilon$ ) such that for every $1 \leq i \leq k$ a sample of $r$ random values from $B\left(p_{i}\right)$ gives us a tuple $\mathbf{q} \in R$ which is a good estimate of $d^{\mathbf{w}}(\mathbf{q}, \mathbf{p})$.

Lemma 3 (Sampling Lemma). There exists $c>0$ such that for every $\epsilon>0$, positive integers $k$ and $r \geq c \cdot \frac{k}{\epsilon^{2}} \cdot \log \frac{1}{\epsilon}, k$-tuples $\mathbf{p}=\left(p_{1}, \ldots, p_{k}\right)$ with $0 \leq p_{i} \leq 1$, and $\mathbf{w}=\left(w_{1}, \ldots, w_{k}\right)$ with $0 \leq w_{i}$, and relation $R \subseteq\{0,1\}^{k}$, the following is satisfied.

For every $1 \leq i \leq k$ and $1 \leq j \leq r$, let $X_{i}^{j} \sim B\left(p_{i}\right)$, and let $\mathbf{Q}=\left(Q_{1}, \ldots Q_{k}\right)$ be the $k$-tuple of random variables, where $Q_{i}=\frac{1}{r} \sum_{j=1}^{r} X_{i}^{j}$. Let $d_{\text {min }}$ be the minimum distance weighted by $\mathbf{w}$ from $\mathbf{p}$ to a $k$-tuple from $R$. Let $\mathbf{q}$ be a $k$-tuple from $R$ within the minimum weighted by $\mathbf{w}$ distance to $\mathbf{Q}$, that is, $\mathbf{q}=\operatorname{argmin}_{\mathbf{x} \in R} d^{\mathbf{w}}(\mathbf{x}, \mathbf{Q})$, and let $D=d^{\mathbf{w}}(\mathbf{q}, \mathbf{p})$. Then $\mathbf{E}[D] \leq(1+\epsilon) d_{\text {min }}$.

Proof. Let $\mathbf{u}=\operatorname{argmin}_{\mathbf{x} \in R} d^{\mathbf{w}}(\mathbf{x}, \mathbf{p})$. Then $d_{\text {min }}=d^{\mathbf{w}}(\mathbf{u}, \mathbf{p})$. Let $R_{\text {small }}$ be the set of all tuples $\mathbf{v} \in R$ such that $d^{\mathbf{w}}(\mathbf{v}, \mathbf{p}) \leq\left(1+\frac{\epsilon}{2}\right) d_{\text {min }}$. Let $R_{\text {big }}=R \backslash R_{\text {small }}$. We will prove the following claim.

Claim 1. For every $\mathbf{v} \in R_{b i g}$,

$$
\operatorname{Pr}\left(d^{\mathbf{w}}(\mathbf{v}, \mathbf{Q}) \leq d^{\mathbf{w}}(\mathbf{u}, \mathbf{Q})\right) \leq \frac{d_{\min }}{d^{\mathbf{w}}(\mathbf{v}, \mathbf{p})} \cdot \frac{\epsilon}{2^{k+1}}
$$

Assuming Claim 1 we complete the proof of the lemma:

$$
\begin{array}{r}
\mathbf{E}[D]=\sum_{v \in R_{\text {small }}} d^{\mathbf{w}}(\mathbf{v}, \mathbf{p}) \cdot \operatorname{Pr}(\mathbf{q}=\mathbf{v})+\sum_{v \in R_{\text {big }}} d^{\mathbf{w}}(\mathbf{v}, \mathbf{p}) \cdot \operatorname{Pr}(\mathbf{q}=\mathbf{v}) \\
\leq d_{\text {min }}\left(1+\frac{\epsilon}{2}\right)+\sum_{v \in R_{\text {big }}} d^{\mathbf{w}}(\mathbf{v}, \mathbf{p}) \cdot \operatorname{Pr}\left(d^{\mathbf{w}}(\mathbf{v}, \mathbf{Q}) \leq d^{\mathbf{w}}(\mathbf{u}, \mathbf{Q})\right) \\
\leq d_{\text {min }}\left(1+\frac{\epsilon}{2}\right)+\sum_{v \in R_{\text {big }}} d^{\mathbf{w}}(\mathbf{v}, \mathbf{p}) \cdot \frac{d_{\text {min }}}{d^{\mathbf{w}}(\mathbf{v}, \mathbf{p})} \cdot \frac{\epsilon}{2^{k+1}} \\
\leq d_{\text {min }}\left(1+\frac{\epsilon}{2}\right)+d_{\text {min }} \cdot \frac{\epsilon}{2} \leq d_{\text {min }}(1+\epsilon) .
\end{array}
$$

Hence, all that remains to prove the lemma is to prove Claim 1.

Proof of Claim 1. We will assume without loss of generality that $\epsilon \leq \frac{1}{10}$. By renaming 0 to 1 and vice versa at the coordinates $i$ where $u_{i}=1$, we may assume that $\mathbf{u}=\mathbf{0}$. Thus $d_{\text {min }}=d^{\mathbf{w}}(\mathbf{0}, \mathbf{p})$. We may now rewrite the statement of the claim as:

$$
\operatorname{Pr}\left(d^{\mathbf{w}}(\mathbf{v}, \mathbf{Q}) \leq d^{\mathbf{w}}(\mathbf{0}, \mathbf{Q})\right) \leq \frac{d^{\mathbf{w}}(\mathbf{0}, \mathbf{p})}{d^{\mathbf{w}}(\mathbf{v}, \mathbf{p})} \cdot \frac{\epsilon}{2^{k+1}} .
$$

Consider now the weight $k$-tuple $\mathbf{w}^{\prime}=\left(w_{1}^{\prime}, \ldots, w_{k}^{\prime}\right)$ where $w_{i}^{\prime}=w_{i}$ if $v_{i}=1$ and $w_{i}^{\prime}=0$ if $v_{i}=0$. We have that $\operatorname{Pr}\left(d^{\mathbf{w}}(\mathbf{v}, \mathbf{Q}) \leq d^{\mathbf{w}}(\mathbf{0}, \mathbf{Q})\right)=\operatorname{Pr}\left(d^{\mathbf{w}^{\prime}}(\mathbf{v}, \mathbf{Q}) \leq d^{\mathbf{w}^{\prime}}(\mathbf{0}, \mathbf{Q})\right)$, and that $\frac{d^{\mathbf{w}^{\prime}}(\mathbf{0}, \mathbf{p})}{d^{\mathbf{w}^{\prime}}(\mathbf{v}, \mathbf{p})} \leq \frac{d^{\mathbf{w}}(\mathbf{0}, \mathbf{p})}{d^{\mathbf{w}}(\mathbf{v}, \mathbf{p})}$. Hence, in order to prove (1), it is sufficient to prove 


$$
\operatorname{Pr}\left(d^{\mathbf{w}^{\prime}}(\mathbf{v}, \mathbf{Q}) \leq d^{\mathbf{w}^{\prime}}(\mathbf{0}, \mathbf{Q})\right) \leq \frac{d^{\mathbf{w}^{\prime}}(\mathbf{0}, \mathbf{p})}{d^{\mathbf{w}^{\prime}}(\mathbf{v}, \mathbf{p})} \cdot \frac{\epsilon}{2^{k+1}}
$$

In other words, it is sufficient to prove (1) under the additional assumption that $w_{i}=$ 0 whenever $v_{i}=0$. Under this assumption we have that $d^{\mathbf{w}}(\mathbf{v}, \mathbf{Q})=d^{\mathbf{w}}(\mathbf{1}, \mathbf{Q})$, and that $d^{\mathbf{w}}(\mathbf{v}, \mathbf{p})=d^{\mathbf{w}}(\mathbf{1}, \mathbf{p})$. Thus, it is suffices to prove that $d^{\mathbf{w}}(\mathbf{1}, \mathbf{p}) \geq\left(1+\frac{\epsilon}{2}\right) \cdot d^{\mathbf{w}}(\mathbf{0}, \mathbf{p})$ implies that

$$
\operatorname{Pr}\left(d^{\mathbf{w}}(\mathbf{1}, \mathbf{Q}) \leq d^{\mathbf{w}}(\mathbf{0}, \mathbf{Q})\right) \leq \frac{d^{\mathbf{w}}(\mathbf{0}, \mathbf{p})}{d^{\mathbf{w}}(\mathbf{1}, \mathbf{p})} \cdot \frac{\epsilon}{2^{k+1}}
$$

Let $w^{*}=\sum_{i=1}^{k} w_{i}$. We have that $d^{\mathbf{w}}(\mathbf{0}, \mathbf{p})+d^{\mathbf{w}}(\mathbf{1}, \mathbf{p})=w^{*}$. Thus $d^{\mathbf{w}}(\mathbf{1}, \mathbf{p}) \geq\left(1+\frac{\epsilon}{2}\right) \cdot d^{\mathbf{w}}(\mathbf{0}, \mathbf{p})$ implies that $d^{\mathbf{w}}(\mathbf{0}, \mathbf{p}) \leq \frac{w^{*}}{2+\frac{\epsilon}{2}}$. We also have that $d^{\mathbf{w}}(\mathbf{1}, \mathbf{Q})+d^{\mathbf{w}}(\mathbf{0}, \mathbf{Q})=w^{*}$, and therefore $d^{\mathbf{w}}(\mathbf{1}, \mathbf{Q}) \leq d^{\mathbf{w}}(\mathbf{0}, \mathbf{Q})$ if and only if $d^{\mathbf{w}}(\mathbf{0}, \mathbf{Q}) \geq \frac{w^{*}}{2}$. Furthermore, $d^{\mathbf{w}}(\mathbf{1}, \mathbf{p}) \leq w^{*}$. Hence, to prove the claim (in partiular Equation 2) it is sufficient to show that $d^{\mathbf{w}}(\mathbf{0}, \mathbf{p}) \leq \frac{w^{*}}{2+\frac{\epsilon}{2}}$ implies

$$
\operatorname{Pr}\left(d^{\mathbf{w}}(\mathbf{0}, \mathbf{Q}) \geq \frac{w^{*}}{2}\right) \leq \frac{d^{\mathbf{w}}(\mathbf{0}, \mathbf{p})}{w^{*}} \cdot \frac{\epsilon}{2^{k+1}} .
$$

We now prove Equation 3 distinguishing between two cases.

Case $1, d^{\mathbf{w}}(\mathbf{0}, \mathbf{p})>\frac{w^{*}}{128 k}$.

We have that $d^{\mathbf{w}}(\mathbf{0}, \mathbf{Q})=\sum_{i=1}^{k} \sum_{j=1}^{r} \frac{w_{i}}{r} X_{i}^{j}$. Thus, $d^{\mathbf{w}}(\mathbf{0}, \mathbf{Q})$ is the sum of $k r$ independent random variables, grouped into groups of size $r$, where all variables in group $i$ take value $\frac{w_{i}}{r}$ with probability $p_{i}$ and value 0 with probability $1-p_{i}$. It follows that $\mathbf{E}\left[d^{\mathbf{w}}(\mathbf{0}, \mathbf{Q})\right]=d^{\mathbf{w}}(\mathbf{0}, \mathbf{p}) \leq \frac{w^{*}}{2+\frac{\epsilon}{2}}$. Hence $\frac{w^{*}}{2}-\mathbf{E}\left[d^{\mathbf{w}}(\mathbf{0}, \mathbf{Q})\right] \geq \frac{w^{*}}{2}-\frac{w^{*}}{2+\frac{\epsilon}{2}}>\frac{\epsilon w^{*}}{10}$, where the last inequality follows from the assumption that $\epsilon \leq \frac{1}{10}$. Thus we may use Proposition 4 to upper bound $\operatorname{Pr}\left(d^{\mathbf{w}}(\mathbf{0}, \mathbf{Q}) \geq \frac{w^{*}}{2}\right)$.

$$
\begin{array}{r}
\operatorname{Pr}\left(d^{\mathbf{w}}(\mathbf{0}, \mathbf{Q}) \geq \frac{w^{*}}{2}\right) \leq \operatorname{Pr}\left(d^{\mathbf{w}}(\mathbf{0}, \mathbf{Q})-\mathbf{E}\left[d^{\mathbf{w}}(\mathbf{0}, \mathbf{Q})\right]>\frac{\epsilon w^{*}}{10}\right) \leq \exp \left(-\frac{2 \epsilon^{2}\left(w^{*}\right)^{2}}{100 \Sigma_{i=1}^{k} \Sigma_{j=1}^{r}\left(\frac{w_{i}}{r}\right)^{2}}\right) \\
\leq \exp \left(-\frac{\epsilon^{2} r}{50}\right) \leq \frac{w^{*}}{128 k} \cdot \frac{128 k}{w^{*}} \cdot \frac{\epsilon}{128 k \cdot 2^{k+1}} \leq \frac{d^{\mathbf{w}}(\mathbf{0}, \mathbf{p})}{w^{*}} \cdot \frac{\epsilon}{2^{k+1}} .
\end{array}
$$

Here the second transition is by Proposition 4, while the fourth is by the choice of $r=\Omega\left(\frac{k}{\epsilon^{2}}\right.$. $\left.\log \frac{1}{\epsilon}\right)$.

Case $2, d^{\mathbf{w}}(\mathbf{0}, \mathbf{p}) \leq \frac{w^{*}}{128 k}$.

For every $i$ such that $w_{i} \geq \frac{w^{*}}{4 k}$, we have $p_{i} \leq \frac{1}{32}$. Since $Q_{i}$ is binomially distributed we have that

$$
\operatorname{Pr}\left(Q_{i} \geq \frac{1}{4}\right)=\sum_{t=\left\lceil\frac{r}{4}\right\rceil}^{r}\left(\begin{array}{l}
r \\
t
\end{array}\right) p_{i}^{t}\left(1-p_{i}\right)^{r-t} \leq 2^{r} \cdot p_{i}^{\frac{r}{4}} \leq p_{i} \cdot 2^{r} \cdot\left(\frac{1}{32}\right)^{\frac{r}{4}-1} \leq p_{i} \cdot 2^{-\frac{r}{4}-5} \leq \frac{p_{i}}{4 k^{2}} \cdot \frac{\epsilon}{2^{k+1}}
$$

Here the last inequality follows from the fact that of $r=\Omega\left(\frac{k}{\epsilon^{2}} \cdot \log \frac{1}{\epsilon}\right)$

For every $i$ such that $w_{i} \geq \frac{w^{*}}{4 k}$ we have that $p_{i} \leq \frac{d^{\mathbf{w}}(\mathbf{0}, \mathbf{p})}{w^{*}} \cdot 4 k$, since otherwise $w_{i} p_{i}>d^{\mathbf{w}}(\mathbf{0}, \mathbf{p})$, a contradiction. Thus, for every such $i$ we have

$$
\operatorname{Pr}\left(Q_{i} \geq \frac{1}{4}\right) \leq \frac{d^{\mathbf{w}}(\mathbf{0}, \mathbf{p})}{w^{*} k} \cdot \frac{\epsilon}{2^{k+1}} .
$$


By the union bound, we have that

$$
\operatorname{Pr}\left(\sum_{i: w_{i} \geq \frac{w^{*}}{4 k}} w_{i} Q_{i} \geq \frac{w^{*}}{4}\right) \leq \frac{d^{\mathbf{w}}(\mathbf{0}, \mathbf{p})}{w^{*}} \cdot \frac{\epsilon}{2^{k+1}} .
$$

Since

$$
\sum_{i: w_{i}<\frac{w^{*}}{4 k}} w_{i} Q_{i}<\frac{w^{*}}{4}
$$

(with probability 1), it follows that

$$
\operatorname{Pr}\left(d^{\mathbf{w}}(\mathbf{0}, \mathbf{Q}) \geq \frac{w^{*}}{2}\right) \leq \frac{d^{\mathbf{w}}(\mathbf{0}, \mathbf{p})}{w^{*}} \cdot \frac{\epsilon}{2^{k+1}} .
$$

This proves the claim, and completes the proof of Lemma 3.

\section{Warm up: Deterministic PTAS}

As a warm up, let us show how Sampling Lemma can be used to obtain a deterministic PTAS for Binary Constrained Clustering. Towards that we need the following definition.

Definition 8. Let $k, m \in \mathbb{N}$ and $\mathcal{R}=\left\{R_{1}, \ldots, R_{m}\right\}$ be a family of relations, where $R_{i} \subseteq\{0,1\}^{k}$ for each $i \in\{1, \ldots, m\}$. Let $\left\{S_{1}, \ldots, S_{k}\right\}$ be a family of multisets of vectors from $\{0,1\}^{m}$ and $w_{1}, \ldots, w_{k} \in \mathbb{R}_{\geq 0}$. For a vector (multi) set $B \subseteq\{0,1\}^{m}$, let $z^{(i)}(B)$ be the number of vectors in $B$ with the $i$-th entry equal to 0 and let $d^{(i)}(B)=|B|-z^{(i)}(B)$ be the number of vectors in $B$ with the $i$-th entry equal to 1 . Then best $_{\mathcal{R}}\left(S_{1}, \ldots, S_{k}, w_{1}, \ldots, w_{k}\right)$ is a set of vectors $\left\{\mathbf{c}_{1}, \ldots, \mathbf{c}_{k}\right\}$ satisfying $\mathcal{R}$ which is defined as follows. For $i \in\{1, \ldots, m\}$ and a $k$-tuple $\mathbf{b}=\left(b_{1}, \ldots, b_{k}\right) \in R_{i}$, let

$$
f_{i}\left(b_{1}, \ldots, b_{k}\right)=\sum_{j \in I_{b}} w_{j} \cdot z^{(i)}\left(S_{j}\right)+\sum_{j \in[k] \backslash I_{b}} w_{j} \cdot d^{(i)}\left(S_{j}\right),
$$

where $I_{b}=\left\{j \in\{1, \ldots, k\}: b_{j}=1\right\}$. The set $\left\{\mathbf{c}_{1}, \ldots, \mathbf{c}_{k}\right\}$ be such that $\left(\mathbf{c}_{1}[i], \ldots, \mathbf{c}_{k}[i]\right) \in R_{i}$ and $f_{i}\left(\mathbf{c}_{1}[i], \ldots, \mathbf{c}_{k}[i]\right)=\min _{\mathbf{b} \in R_{i}} f_{i}(\mathbf{b}), 1 \leq i \leq m$.

Lemma 4. Let $J=\left(X, k, \mathcal{R}=\left\{R_{1}, \ldots, R_{m}\right\}\right)$ of Binary Constrained Clustering, $\varepsilon>0$ and $r=\Theta\left(\frac{k}{\varepsilon^{2}} \log \frac{1}{\varepsilon}\right)$ is the constant defined in Lemma 3. Then there exist $w_{1}, \ldots, w_{k} \in \mathbb{N}$ and a family $\left\{S_{1}^{\prime}, \ldots, S_{k}^{\prime}\right\}$ of $r$ sized multisets of vectors from $X$, such that

$$
\operatorname{cost}\left(X, \operatorname{best}_{\mathcal{R}}\left(S_{1}^{\prime}, \ldots, S_{k}^{\prime}, w_{1}, \ldots, w_{k}\right)\right) \leq(1+\varepsilon) \mathrm{OPT}(J) .
$$

Proof. Let $C^{*}=\left\{\mathbf{c}_{1}^{*}, \ldots, \mathbf{c}_{k}^{*}\right\}$ be an optimal solution to $J$ with corresponding clusters $P_{1}, \ldots, P_{k}$. That is $\mathrm{OPT}(J)=\operatorname{cost}\left(X, C^{*}\right)=\sum_{i=1}^{k} \operatorname{cost}\left(P_{i},\left\{c_{i}^{*}\right\}\right)$. For each $i \in\{1, \ldots, k\}$, we set $w_{i}=\left|P_{i}\right|$. For each $i \in\{1, \ldots, k\}$, we define a multiset $S_{i}$ of $r$ vectors, where each vector in $S_{i}$ is chosen uniformly at random with repetition from $P_{i}$. To prove the lemma it is enough to prove that

$$
\mathbf{E}\left[\operatorname{cost}\left(X, \operatorname{best}_{\mathcal{R}}\left(S_{1}, \ldots, S_{k}, w_{1}, \ldots, w_{k}\right)\right)\right] \leq(1+\varepsilon) \mathrm{OPT}(J)
$$

Recall the definition of functions $f_{i}, 1 \leq i \leq m$ (see Definition 8). For $i \in\{1, \ldots, m\}$ and a $k$-tuple $\mathbf{b}=\left(b_{1}, \ldots, b_{k}\right) \in R_{i}$,

$$
f_{i}\left(b_{1}, \ldots, b_{k}\right)=\sum_{j \in I_{b}} w_{j} \cdot z^{(i)}\left(S_{j}\right)+\sum_{j \in[k] \backslash I_{b}} w_{j} \cdot d^{(i)}\left(S_{j}\right)
$$


where $I_{b}=\left\{j \in\{1, \ldots, k\}: b_{j}=1\right\}$. The set $\operatorname{best}_{\mathcal{R}}\left(S_{1}, \ldots, S_{k}, w_{1}, \ldots, w_{k}\right)=\left\{\mathbf{c}_{1}, \ldots, \mathbf{c}_{k}\right\}$ be such that $\left(\mathbf{c}_{1}[i], \ldots, \mathbf{c}_{k}[i]\right) \in R_{i}$ and $f_{i}\left(\mathbf{c}_{1}[i], \ldots, \mathbf{c}_{k}[i]\right)=\min _{\mathbf{b} \in R_{i}} f_{i}(\mathbf{b}), 1 \leq i \leq m$.

Notice that best $\mathcal{R}_{\mathcal{R}}\left(S_{1}, \ldots, S_{k}, w_{1}, \ldots, w_{k}\right)=\left\{\mathbf{c}_{1}, \ldots, \mathbf{c}_{k}\right\}$ is a random variable. We define a random variable $Y=\sum_{i=1}^{k} \operatorname{cost}\left(P_{i},\left\{\mathbf{c}_{i}\right\}\right)$. To prove (4), it is enough prove that $\mathbf{E}[Y] \leq$ $(1+\varepsilon) \mathrm{OPT}(J)$. We define functions $g_{i}$ for all $i \in[m]$, which denotes the cost of each element in the relation $R_{i}$ with respect to the partition $P_{1} \uplus \ldots \uplus P_{k}$ of $X$. Formally, for each $\mathbf{b}=$ $\left(b_{1}, \ldots, b_{k}\right) \in R_{i}$, we put

$$
g_{i}(\mathbf{b})=\sum_{j \in I_{b}} z^{(i)}\left(P_{j}\right)+\sum_{j \in[k] \backslash I_{b}} d^{(i)}\left(P_{j}\right),
$$

where $I_{b}=\left\{j \in\{1, \ldots, k\}: b_{j}=1\right\}$. Let $V_{i}=g_{i}\left(\mathbf{c}_{1}^{*}[i], \ldots, \mathbf{c}_{k}^{*}[i]\right)$. Notice that $\operatorname{OPT}(J)=$ $\sum_{i=1}^{m} V_{i}$. Let $Y_{i}=g_{i}\left(\mathbf{c}_{1}[i], \ldots, \mathbf{c}_{k}[i]\right), 1 \leq i \leq m$. By (6) and the definition of $Y$, we have that $Y=\sum_{i=1}^{m} Y_{i}$. By the linearity of expectation, we have that $\mathbf{E}[Y]=\sum_{i=1}^{m} \mathbf{E}\left[Y_{i}\right]$. Thus, to prove that $\mathbf{E}[Y] \leq(1+\varepsilon) \mathrm{OPT}(J)$, it is sufficient to prove that $\mathbf{E}\left[Y_{i}\right] \leq(1+\varepsilon) V_{i}$ for every $i \in[m]$.

Claim 2. For every $i \in[m], \mathbf{E}\left[Y_{i}\right] \leq(1+\varepsilon) V_{i}$.

Proof. Fix an index $i \in[m]$. Let $z_{j}=z^{(i)}\left(P_{j}\right)$ and $d_{j}=d^{(i)}\left(P_{j}\right)$. Thus $z_{j}\left(d_{j}\right)$ is the number of vectors from $P_{j}$ whose $i$-th coordinate is $0(1)$. Let $n_{j}=\left|P_{j}\right|$ and $p_{j}=\frac{d_{j}}{n_{j}}, j \in\{1, \ldots, k\}$. Since each vector from $S_{j}$ is equally likely to be any vector in $P_{j}$, for every vector $v \in S_{j}$,

$$
\operatorname{Pr}(v[i]=1)=\frac{d_{j}}{n_{j}}=p_{j} \quad \text { and } \quad \operatorname{Pr}(v[i]=0)=\frac{z_{j}}{n_{j}}=1-p_{j} .
$$

Let $\mathbf{p}=\left(p_{1}, \ldots, p_{k}\right)$. We define $k$-tuple $\mathbf{w}=\left(w_{1}, \ldots, w_{k}\right)$. and claim that for every $\mathbf{b}=$ $\left(b_{1}, \ldots, b_{k}\right) \in R_{i}, d^{\mathbf{y}}(\mathbf{b}, \mathbf{p})=g_{i}(\mathbf{b})$. Indeed,

$$
\begin{aligned}
d^{\mathbf{w}}(\mathbf{b}, \mathbf{p}) & =\sum_{j=1}^{k} w_{j}\left|b_{j}-p_{j}\right| \\
& =\sum_{j \in I_{b}} w_{j}\left(1-p_{j}\right)+\sum_{j \in[k] \backslash I_{b}} w_{j} \cdot p_{j} \\
& =\sum_{j \in I_{b}} z_{j}+\sum_{j \in[k] \backslash I_{b}} d_{j} \\
& =g_{i}(\mathbf{b}) .
\end{aligned}
$$

For each set $S_{j}=\left\{v_{1}, \ldots, v_{r}\right\}, 1 \leq j \leq k$ and $1 \leq q \leq r$, we define random variable $X_{j}^{q}$ which is 1 when $v_{q}[i]=1$ and 0 otherwise. By (7), we have that $X_{j}^{q} \sim B\left(p_{j}\right)$ for all $1 \leq j \leq k$ and $1 \leq q \leq r$. Let $\mathbf{Q}=\left(Q_{1}, \ldots Q_{k}\right)$ be the $k$-tuple of random variables, where $Q_{j}=\frac{1}{r} \sum_{q=1}^{r} X_{j}^{q}$. From the definitions of $z^{(i)}\left(S_{j}\right), d^{(i)}\left(S_{j}\right)$ and $X_{j}^{q}$, we have that

$$
z^{(i)}\left(S_{j}\right)=\sum_{q=1}^{r}\left(1-X_{j}^{q}\right) \quad \text { and } \quad d^{(i)}\left(S_{j}\right)=\sum_{q=1}^{r} X_{j}^{q} .
$$


Let $\mathbf{w}=\left(w_{1}, \ldots, w_{k}\right)$. For $\mathbf{b} \in R_{i}$, we upper bound $d^{\mathbf{w}}(\mathbf{b}, \mathbf{Q})$ in terms of $f_{i}(\mathbf{b})$.

$$
\begin{aligned}
d^{\mathbf{w}}(\mathbf{b}, \mathbf{Q}) & =\sum_{j=1}^{k} w_{j}\left|b_{i}-Q_{j}\right| \\
& \leq \sum_{j \in I_{b}} w_{j}\left(1-Q_{j}\right)+\sum_{j \in[k] \backslash I_{b}} w_{j} \cdot Q_{j} \\
& \leq \sum_{j \in I_{b}} w_{j} \cdot\left(1-\frac{1}{r} \sum_{q=1}^{r} X_{j}^{q}\right)+\sum_{j \in[k] \backslash I_{b}} w_{j} \cdot\left(\frac{1}{r} \sum_{q=1}^{r} X_{j}^{q}\right) \\
& \leq \frac{1}{r}\left(\sum_{j \in I_{b}} w_{j} \cdot\left(\sum_{q=1}^{r}\left(1-X_{j}^{q}\right)\right)+\sum_{j \in[k] \backslash I_{b}} w_{j} \cdot\left(\sum_{q=1}^{r} X_{j}^{q}\right)\right) \\
& \leq \frac{1}{r}\left(\sum_{j \in I_{b}} w_{j} \cdot z^{(i)}\left(S_{j}\right)+\sum_{j \in[k] \backslash I_{b}} w_{j} \cdot d^{(i)}\left(S_{j}\right)\right) \\
& \leq \frac{1}{r} f_{i}(\mathbf{b}) .
\end{aligned}
$$

Let

$$
\mathbf{q}=\operatorname{argmin}_{\mathbf{x} \in R_{i}} d^{\mathbf{w}}(\mathbf{x}, \mathbf{Q}) .
$$

By (19) and by the definition of the vector set $\left\{\mathbf{c}_{1}, \ldots, \mathbf{c}_{k}\right\}$, we have that $\mathbf{q}=\left(\mathbf{c}_{1}[i], \ldots, \mathbf{c}_{k}[i]\right)$.

We also define $k$-tuple

$$
\mathbf{u}=\operatorname{argmin}_{\mathbf{x} \in R_{i}} d^{\mathbf{w}}(\mathbf{x}, \mathbf{p}) .
$$

This implies that

$$
d^{\mathbf{w}}(\mathbf{u}, \mathbf{p})=g_{i}\left(\mathbf{c}_{1}^{*}[i], \ldots, \mathbf{c}_{k}^{*}[k]\right)=V_{i} .
$$

Thus the minimum weighted by $\mathbf{w}$ distance $d_{\text {min }}$ from $\mathbf{p}$ to a $k$-tuple from $R_{i}$ is equal to $d^{\mathbf{w}}(\mathbf{u}, \mathbf{p})$. Let $D$ be the random variable which is a minimum weighted by $\mathbf{w}$ distance from $\mathbf{q}$ to p. By Lemma 3,

$$
\mathbf{E}\left[d^{\mathbf{w}}(\mathbf{q}, \mathbf{p})\right]=\mathbf{E}[D] \leq(1+\epsilon) d_{\min }=(1+\epsilon) d^{\mathbf{w}}(\mathbf{u}, \mathbf{p}) .
$$

Finally we upper bound $\mathbf{E}\left[Y_{i}\right]$.

$$
\begin{aligned}
\mathbf{E}\left[Y_{i}\right] & =\mathbf{E}\left[g_{i}(\mathbf{q})\right] \\
& =\mathbf{E}\left[d^{\mathbf{w}}(\mathbf{q}, \mathbf{p})\right] \\
& \leq(1+\varepsilon) \cdot d^{\mathbf{w}}(\mathbf{u}, \mathbf{p}) \\
& \leq(1+\varepsilon) V_{i} .
\end{aligned}
$$

This completes the proof of the claim.

By Claim 3, the fact that $\mathrm{OPT}(J)=\sum_{i=1}^{m} V_{i}$ and by the linearity of expectation, we have that $\mathbf{E}[Y] \leq(1+\varepsilon) \mathrm{OPT}(J)$. This completes the proof of the lemma.

Lemma 4 implies Theorem 1. The remaining part of the paper is built towards obtaining a linear time randomized approximation scheme for BINARY CONSTRAIned Clustering. 


\section{Sampling instances with large clusters}

In this section we prove the algorithmic variant of Sampling Lemma which will be the main engine of our randomized algorithm. Informally, the lemma says that if there is an optimal solution $C$ for the set of vectors $X$ such that each of the clusters corresponding to $C$ contains a large fraction of the vectors from $X$, then sampling constantly many vectors from $X$ for each cluster is a good estimate for a good approximate solution. In fact, we need a stronger property: We want to derive a good clustering of a subset of vectors $Z \subseteq X$ which is unknown to us (a hidden subset).

Lemma 5 (Algorithmic Sampling Lemma). Let $X \subseteq\{0,1\}^{m}$ be a set of $n$ binary vectors and $Z \subseteq X$ be (an unknown) set of vectors. Let $J=\left(Z, k, \mathcal{R}=\left\{R_{1}, \ldots, R_{m}\right\}\right)$ be an instance of BINary Constrained Clustering. Suppose that there exists a solution $C^{*}=\left\{\mathbf{c}_{1}^{*}, \ldots, \mathbf{c}_{k}^{*}\right\}$ (not necessarily optimal) to $J$ with corresponding clusters $P_{1}, \ldots, P_{k}$ and $1 \geq \beta>0$ such that $\left|P_{j}\right| \geq$ $n \beta$ for all $j \in\{1, \ldots, k\}$. We denote the cost of $C^{*}$ by $V=\operatorname{cost}\left(Z, C^{*}\right)=\sum_{i=1}^{k} \operatorname{cost}\left(P_{i},\left\{c_{i}^{*}\right\}\right)$.

Then there exists an algorithm $\mathcal{A}$ with the following specifications.

- Input of $\mathcal{A}$ is $X, k, \mathcal{R}=\left\{R_{1}, \ldots, R_{m}\right\}, \delta, \varepsilon>0,0<\beta \leq 1$, and values $w_{1}, \ldots, w_{k}$ (promised bounds on the sizes of clusters $P_{i}$ ) such that for some constant $c$, for each $j \in\{1, \ldots, k\}$,

$$
\frac{\left|P_{j}\right|}{c} \leq w_{j} \leq \frac{(1+\delta)\left|P_{j}\right|}{c} .
$$

- Output of $\mathcal{A}$ is a solution $C=\left\{c_{1}, \ldots, c_{k}\right\}$ to $J$ such that $\sum_{i=1}^{k} \operatorname{cost}\left(P_{i},\left\{c_{i}\right\}\right) \leq(1+\varepsilon)^{2}(1+$ $\delta) V$ with probability at least $\frac{\varepsilon \cdot \beta^{r \cdot k}}{1+\varepsilon}$, where $r=\Theta\left(\frac{k}{\varepsilon^{2}} \log \frac{1}{\varepsilon}\right)$, and

- $\mathcal{A}$ runs in time $\mathcal{O}\left(\left(\frac{k}{\varepsilon}\right)^{2} \log \frac{1}{\varepsilon} \cdot \sum_{i=1}^{m}\left|R_{i}\right|\right)$.

Proof. Let $r$ be the constant defined for $k$ and $\varepsilon$ in Lemma 3. That is $r=\Theta\left(\frac{k}{\varepsilon^{2}} \cdot \log \frac{1}{\varepsilon}\right)$. For vector $\mathbf{b}=\left(b_{1}, \ldots, b_{k}\right) \in\{0,1\}^{k}$, we define $I_{b}=\left\{j \in\{1, \ldots, k\}: b_{j}=1\right\}$. For vector set $B \subseteq X$, let $z^{(i)}(B)$ be the number of vectors in $B$ with the $i$-th entry equal to 0 . Similarly, let $d^{(i)}(B)=|B|-z^{(i)}(B)$ be the number of vectors in $B$ with the $i$-th entry equal to 1 .

Algorithm. The algorithm $\mathcal{A}$ is very simple. We sample $k$ times (with possible repetitions) uniformly at random $r$ vectors from $X$. Thus we obtain $k$ sets of vectors $S_{1}, \ldots, S_{k}$, each of the sets is of size $r$. Based on these samples we output solution $C=\left\{\mathbf{c}_{1}, \ldots, \mathbf{c}_{k}\right\}$ as follows. For $i \in\{1, \ldots, m\}$ and a $k$-tuple $\mathbf{b}=\left(b_{1}, \ldots, b_{k}\right) \in R_{i}$, let

$$
f_{i}\left(b_{1}, \ldots, b_{k}\right)=\sum_{j \in I_{b}} w_{j} \cdot z^{(i)}\left(S_{j}\right)+\sum_{j \in[k] \backslash I_{b}} w_{j} \cdot d^{(i)}\left(S_{j}\right) .
$$

Then $C$ is the set of vectors minimizing functions $f_{i}$ subject to constraints $\mathcal{R}$. More precisely, we define a vector set $C=\left\{\mathbf{c}_{1}, \ldots, \mathbf{c}_{k}\right\}$ such that $\left(\mathbf{c}_{1}[i], \ldots, \mathbf{c}_{k}[i]\right) \in R_{i}$ and $f_{i}\left(\mathbf{c}_{1}[i], \ldots, \mathbf{c}_{k}[i]\right)=$ $\min _{\mathbf{b} \in R_{i}} f_{i}(\mathbf{b}), 1 \leq i \leq m$. In other words, $C=\operatorname{best}_{\mathcal{R}}\left(S_{1}, \ldots, S_{k}, w_{1}, \ldots, w_{k}\right)$. Clearly, $C$ is a solution to $J$.

Running time. We assume that input vectors $X$ are stored in an array and that we can sample a vector u.a.r from a set of $n$ vectors stored in an array in constant time. For each $i \in[m]$ and $\mathbf{b} \in R_{i}$, the computation of $f_{i}(\mathbf{b})$ takes time $\mathcal{O}(r \cdot k)$. Then computations of functions $f_{i}(\mathbf{b})$ for all $i \in[m]$ and $b \in R_{i}$ require $\mathcal{O}\left(\left(\sum_{i=1}^{m}\left|R_{i}\right|\right)\left(\frac{k}{\varepsilon}\right)^{2} \log \frac{1}{\varepsilon}\right)$ time. For each $i$, we use an array of length $k$ to store the $k$-tuple from $R_{i}$ which gives the minimum of $f_{i}$ computed so far during the computation. Therefore the running time of the algorithm follows. 
Correctness. Let $\mathscr{E}$ be the event that for all $j \in\{1, \ldots, k\}, S_{j} \subseteq P_{j}$. Since $\left|P_{j}\right| \geq|X| \cdot \beta$ for all $j \in\{1, \ldots, k\}$, we have that

$$
\operatorname{Pr}(\mathscr{E}) \geq \beta^{r \cdot k} .
$$

From now on we assume that the event $\mathscr{E}$ happened. Therefore we can think that each vector in $S_{j}$ is chosen uniformly at random from $P_{j}$ (with repetitions). Notice that the output $C=\left\{\mathbf{c}_{1}, \ldots, \mathbf{c}_{k}\right\}$ is a random variable. We define a random variable $Y=\sum_{i=1}^{k} \operatorname{cost}\left(P_{j},\left\{\mathbf{c}_{j}\right\}\right)$.

Now we prove that $\mathbf{E}[Y \mid \mathscr{E}] \leq(1+\varepsilon)(1+\delta) V$. We define functions $g_{i}$ for all $i \in[m]$, which denotes the cost of each element in the relation $R_{i}$ with respect to the partition $P_{1} \uplus \ldots \uplus P_{k}$ of $Z$. Formally, for each $\mathbf{b}=\left(b_{1}, \ldots, b_{k}\right) \in R_{i}$, we put

$$
g_{i}(\mathbf{b})=\sum_{j \in I_{b}} z^{(i)}\left(P_{j}\right)+\sum_{j \in[k] \backslash I_{b}} d^{(i)}\left(P_{j}\right) .
$$

Let $V_{i}=g_{i}\left(\mathbf{c}_{1}^{*}[i], \ldots, \mathbf{c}_{k}^{*}[i]\right)$. Notice that $V=\sum_{i=1}^{m} V_{i}$. Let $Y_{i}=g_{i}\left(\mathbf{c}_{1}[i], \ldots, \mathbf{c}_{k}[i]\right), 1 \leq i \leq m$. By (15) and the definition of $Y$, we have that $Y=\sum_{i=1}^{m} Y_{i}$. By the linearity of conditional expectation, we have that $\mathbf{E}[Y \mid \mathscr{E}]=\sum_{i=1}^{m} \mathbf{E}\left[Y_{i} \mid \mathscr{E}\right]$. Thus, to prove that $\mathbf{E}[Y \mid \mathscr{E}] \leq$ $(1+\varepsilon)(1+\delta) V$, it is sufficient to prove that $\mathbf{E}\left[Y_{i} \mid \mathscr{E}\right] \leq(1+\varepsilon)(1+\delta) V_{i}$ for every $i \in[m]$.

Claim 3. For every $i \in[m], \mathbf{E}\left[Y_{i} \mid \mathscr{E}\right] \leq(1+\varepsilon)(1+\delta) V_{i}$.

Proof. Fix an index $i \in[m]$. Let $z_{j}=z^{(i)}\left(P_{j}\right)$ and $d_{j}=d^{(i)}\left(P_{j}\right)$. Thus $z_{j}\left(d_{j}\right)$ is the number of vectors from $P_{j}$ whose $i$-th coordinate is $0(1)$. Let $n_{j}=\left|P_{j}\right|$ and $p_{j}=\frac{d_{j}}{n_{j}}, j \in\{1, \ldots, k\}$. Since we assume that $\mathscr{E}$ happened, each vector from $S_{j}$ is equally likely to be any vector in $P_{j}$. That is, for every vector $v \in S_{j}$, we have that

$$
\operatorname{Pr}(v[i]=1 \mid \mathscr{E})=\frac{d_{j}}{n_{j}}=p_{j} \quad \text { and } \quad \operatorname{Pr}(v[i]=0 \mid \mathscr{E})=\frac{z_{j}}{n_{j}}=1-p_{j} .
$$

Let $\mathbf{p}=\left(p_{1}, \ldots, p_{k}\right)$. We define $k$-tuple $\mathbf{y}=\left(n_{1}, \ldots, n_{k}\right)$ and claim that for every $\mathbf{b}=$ $\left(b_{1}, \ldots, b_{k}\right) \in R_{i}, d^{\mathbf{y}}(\mathbf{b}, \mathbf{p})=g_{i}(\mathbf{b})$. Indeed,

$$
\begin{aligned}
d^{\mathbf{y}}(\mathbf{b}, \mathbf{p}) & =\sum_{j=1}^{k} n_{j}\left|b_{j}-p_{j}\right| \\
& =\sum_{j \in I_{b}} n_{j}\left(1-p_{j}\right)+\sum_{j \in[k] \backslash I_{b}} n_{j} \cdot p_{j} \\
& =\sum_{j \in I_{b}} z_{j}+\sum_{j \in[k] \backslash I_{b}} d_{j} \\
& =g_{i}(\mathbf{b}) .
\end{aligned}
$$

For each set $S_{j}=\left\{v_{1}, \ldots, v_{r}\right\}, 1 \leq j \leq k$ and $1 \leq q \leq r$, we define random variable $L_{j}^{q}$ which is 1 when $v_{q}[i]=1$ and 0 otherwise. Let us denote by $X_{j}^{q}$ the random variable $L_{j}^{q} \mid \mathscr{E}$. By (16), we have that $X_{j}^{q} \sim B\left(p_{j}\right)$ for all $1 \leq j \leq k$ and $1 \leq q \leq r$. Let $\mathbf{Q}=\left(Q_{1}, \ldots Q_{k}\right)$ be the $k$-tuple of random variables, where $Q_{j}=\frac{1}{r} \sum_{q=1}^{r} X_{j}^{q}$. From the definitions of $z^{(i)}\left(S_{j}\right), d^{(i)}\left(S_{j}\right)$ and $X_{j}^{q}$, we have that

$$
z^{(i)}\left(S_{j}\right)=\sum_{q=1}^{r}\left(1-X_{j}^{q}\right) \quad \text { and } \quad d^{(i)}\left(S_{j}\right)=\sum_{q=1}^{r} X_{j}^{q} .
$$


Let $\mathbf{w}=\left(w_{1}, \ldots, w_{k}\right)$. For $\mathbf{b} \in R_{i}$, we upper bound $d^{\mathbf{w}}(\mathbf{b}, \mathbf{Q})$ in terms of $f_{i}(\mathbf{b})$.

$$
\begin{aligned}
d^{\mathbf{w}}(\mathbf{b}, \mathbf{Q}) & =\sum_{j=1}^{k} w_{j}\left|b_{i}-Q_{j}\right| \\
& \leq \sum_{j \in I_{b}} w_{j}\left(1-Q_{j}\right)+\sum_{j \in[k] \backslash I_{b}} w_{j} \cdot Q_{j} \\
& \leq \sum_{j \in I_{b}} w_{j} \cdot\left(1-\frac{1}{r} \sum_{q=1}^{r} X_{j}^{q}\right)+\sum_{j \in[k] \backslash I_{b}} w_{j} \cdot\left(\frac{1}{r} \sum_{q=1}^{r} X_{j}^{q}\right) \\
& \leq \frac{1}{r}\left(\sum_{j \in I_{b}} w_{j} \cdot\left(\sum_{q=1}^{r}\left(1-X_{j}^{q}\right)\right)+\sum_{j \in[k] \backslash I_{b}} w_{j} \cdot\left(\sum_{q=1}^{r} X_{j}^{q}\right)\right) \\
& \leq \frac{1}{r}\left(\sum_{j \in I_{b}} w_{j} \cdot z^{(i)}\left(S_{j}\right)+\sum_{j \in[k] \backslash I_{b}} w_{j} \cdot d^{(i)}\left(S_{j}\right)\right) \\
& \leq \frac{1}{r} f_{i}(\mathbf{b}) .
\end{aligned}
$$

Let

$$
\mathbf{q}=\operatorname{argmin}_{\mathbf{x} \in R_{i}} d^{\mathbf{w}}(\mathbf{x}, \mathbf{Q}) .
$$

By (19) and by the definition of the vector set $C=\left\{\mathbf{c}_{1}, \ldots, \mathbf{c}_{k}\right\}$, we have that $\mathbf{q}=\left(\mathbf{c}_{1}[i], \ldots, \mathbf{c}_{k}[i]\right)$.

We also define $k$-tuples

$$
\mathbf{u}=\operatorname{argmin}_{\mathbf{x} \in R_{i}} d^{\mathbf{w}}(\mathbf{x}, \mathbf{p}) \text { and } \mathbf{u}^{*}=\operatorname{argmin}_{\mathbf{x} \in R_{i}} d^{\mathbf{y}}(\mathbf{x}, \mathbf{p}) .
$$

Thus the minimum weighted by $\mathbf{w}$ distance $d_{\min }$ from $\mathbf{p}$ to a $k$-tuple from $R_{i}$ is equal to $d^{\mathbf{w}}(\mathbf{u}, \mathbf{p})$. Let $D$ be the random variable which is a minimum weighted by $\mathbf{w}$ distance from $\mathbf{q}$ to p. By Lemma 3,

$$
\mathbf{E}\left[d^{\mathbf{w}}(\mathbf{q}, \mathbf{p}) \mid \mathscr{E}\right]=\mathbf{E}[D] \leq(1+\epsilon) d_{\min }=(1+\epsilon) d^{\mathbf{w}}(\mathbf{u}, \mathbf{p}) .
$$
that

Since $\left(\mathbf{c}_{1}^{*}[i], \ldots, \mathbf{c}_{k}^{*}[i]\right) \in R_{i}$, and because $d^{\mathbf{y}}(\mathbf{b}, \mathbf{p})=g_{i}(\mathbf{b})$ for each $\mathbf{b} \in R_{i}$ (by (17)), we have

$$
d^{\mathbf{y}}\left(\mathbf{u}^{*}, \mathbf{p}\right) \leq g_{i}\left(\mathbf{c}_{1}^{*}[i], \ldots, \mathbf{c}_{k}^{*}[k]\right)=V_{i} .
$$

Finally we upper bound $\mathbf{E}\left[Y_{i} \mid \mathscr{E}\right]$.

$$
\begin{aligned}
\mathbf{E}\left[Y_{i} \mid \mathscr{E}\right] & =\mathbf{E}\left[g_{i}(\mathbf{q}) \mid \mathscr{E}\right] & & \\
& =\mathbf{E}\left[d^{\mathbf{y}}(\mathbf{q}, \mathbf{p}) \mid \mathscr{E}\right] & & (\text { by }(17)) \\
& \leq c \cdot \mathbf{E}\left[d^{\mathbf{w}}(\mathbf{q}, \mathbf{p}) \mid \mathscr{E}\right] & & \left(\text { because } n_{j} \leq c w_{j} \text { for all } j \in[k]\right) \\
& \leq c \cdot(1+\varepsilon) \cdot d^{\mathbf{w}}(\mathbf{u}, \mathbf{p}) & & (\text { by }(20)) \\
& \leq c \cdot(1+\varepsilon) \cdot d^{\mathbf{w}}\left(\mathbf{u}^{*}, \mathbf{p}\right) & & (\text { by the choice of } \mathbf{u}) \\
& \leq(1+\varepsilon)(1+\delta) d^{\mathbf{y}}\left(\mathbf{u}^{*}, \mathbf{p}\right) & & \left(\text { because } c w_{j} \leq(1+\delta) n_{j} \text { for all } j \in[k]\right) \\
& \leq(1+\varepsilon)(1+\delta) V_{i} . & & (\text { by }(21))
\end{aligned}
$$

This completes the proof of the claim.

By Claim 3, the fact that $V=\sum_{i=1}^{m} V_{i}$ and by the linearity of expectation, we have that

$$
\mathbf{E}[Y \mid \mathscr{E}] \leq(1+\varepsilon)(1+\delta) V .
$$


Combined with Markov's inequality (Proposition 3), this implies that

$$
\operatorname{Pr}\left(Y \geq(1+\varepsilon)^{2}(1+\delta) V \mid \mathscr{E}\right) \leq \frac{1}{1+\varepsilon} .
$$

Therefore

$$
\operatorname{Pr}\left(Y \leq(1+\varepsilon)^{2}(1+\delta) V \mid \mathscr{E}\right) \geq \frac{\varepsilon}{1+\varepsilon}
$$

Finally,

$$
\begin{aligned}
\operatorname{Pr}\left(Y \leq(1+\varepsilon)^{2}(1+\delta) V\right) & \geq \operatorname{Pr}\left(\left(Y \leq(1+\varepsilon)^{2}(1+\delta) V\right) \cap \mathscr{E}\right) \\
& =\operatorname{Pr}\left(Y \leq(1+\varepsilon)^{2}(1+\delta) V \mid \mathscr{E}\right) \cdot \operatorname{Pr}(\mathscr{E}) \\
& \geq \frac{\varepsilon}{1+\varepsilon} \cdot \beta^{r \cdot k} \quad \quad \text { (by (22) and (14)) }
\end{aligned}
$$

This completes the proof of the lemma.

\section{Non-irreducible instances and extendable solutions}

For the algorithm of $k$-MEANS CLUSTERING Kumar et al. [26] used the notion of irreducible instances. We introduce a similar notion for Binary Constrained Clustering.

\subsection{Non-irreducible instances}

Definition 9. Let $J=(X, k, \mathcal{R})$ be an instance of Binary Constrained Clustering, $j \in$ $\{2, \ldots, k\}$ and $\alpha>0$. We say that $J$ is $(j, \alpha)$-irreducible if $\mathrm{OPT}_{j-1}(J) \geq(1+\alpha) \mathrm{OPT}_{j}(J)$.

The following property of irreducible instances plays an important role in our algorithm.

Lemma 6. Let $J=(X, k, \mathcal{R})$ be an instance of Binary Constrained Clustering. For every $0<\epsilon \leq 4$ and $0<\alpha \leq \frac{\epsilon}{8 k}$, the following holds. Let

$$
\widehat{k}=\left\{\begin{array}{l}
1, \text { if } J \text { is not }(i, \alpha) \text {-irreducible for all } i \in\{2, \ldots, k\}, \\
\max \{i \mid J \text { is }(i, \alpha) \text {-irreducible }\}, \text { otherwise. }
\end{array}\right.
$$

Then $\operatorname{OPT}_{\widehat{k}}(J) \leq\left(1+\frac{\epsilon}{4}\right) \mathrm{OPT}(J)$.

Proof. By the choice of $\widehat{k}$, for every $\widehat{k} \leq i<k$, we have that $\operatorname{OPT}_{i}(J) \leq(1+\alpha) \operatorname{OPT}_{i+1}(J)$. Thus $\mathrm{OPT}_{\widehat{k}}(J) \leq(1+\alpha)^{k} \mathrm{OPT}_{k}(J)=(1+\alpha)^{k} \mathrm{OPT}(J)$. Since

$$
\begin{aligned}
(1+\alpha)^{k} & \leq\left(1+\frac{\epsilon}{8 k}\right)^{k}=\sum_{i=0}^{k}\left(\begin{array}{c}
k \\
i
\end{array}\right)\left(\frac{\epsilon}{8 k}\right)^{i} \\
& \leq \sum_{i=0}^{k}\left(\frac{\epsilon}{8}\right)^{i}=\left(1+\frac{\epsilon}{8} \sum_{i=0}^{k-1}\left(\frac{\epsilon}{8}\right)^{i}\right) \\
& \left.\leq\left(1+\frac{\epsilon}{8} \sum_{i=0}^{k-1}\left(\frac{1}{2}\right)^{i}\right) \leq\left(1+\frac{\epsilon}{4}\right), \quad \text { (since } \epsilon \leq 4\right)
\end{aligned}
$$

we have that $\mathrm{OPT}_{\widehat{k}}(J) \leq\left(1+\frac{\epsilon}{4}\right) \operatorname{OPT}(J)$. 
Due to Proposition 5 and Lemma 6 , to obtain an $(1+\epsilon)$-approximate solution to BINARY Constrained Clustering, it is sufficient to learn how to approximate irreducible instances. Indeed, let $J=\left(X, k, \mathcal{R}=\left\{R_{1}, \ldots, R_{k}\right\}\right)$ be an instance to Binary Constrained Clustering and suppose that for $\epsilon>0$ and $\alpha \leq \frac{\epsilon}{8 k}$, instance $J$ is not $(k, \alpha)$-irreducible. Then for the index $\widehat{k}$ defined in Lemma 6 , we have that $\widehat{k}<k$ and $\operatorname{OPT}_{\widehat{k}}(J) \leq\left(1+\frac{\epsilon}{4}\right) \operatorname{OPT}(J)$. The definition of $\operatorname{OPT}_{\widehat{k}}(J)$, implies that

$$
\mathrm{OPT}_{\widehat{k}}(J)=\min \left\{\mathrm{OPT}\left(X, \widehat{k}, \operatorname{proj}_{I}(\mathcal{R})\right) \mid I \subseteq\{1, \ldots, k\} \text { and }|I|=\widehat{k}\right\}
$$

We can make a guess for the value of $\widehat{k}$ and then guess an index subset $I \subset\{1, \ldots, k\}$ of size $\widehat{k}$. For each of the $(k-1) \cdot 2^{k-1}$ guesses of $\widehat{k}$ and $I$, we form instance $J^{\prime}=\left(X, \widehat{k}, \operatorname{proj}_{I}(\mathcal{R})\right)$. We know that for at least one of our guesses, we will have an $(\widehat{k}, \alpha)$-irreducible instance $J^{\prime}$ with $\mathrm{OPT}\left(J^{\prime}\right)=\mathrm{OPT}_{\widehat{k}}(J)$. By Lemma 6 and Proposition 5 , any $(1+\epsilon / 4)$-approximate solution to $J^{\prime}$ is extendable in linear time to a $(1+\epsilon / 4)^{2}$-approximate solution (and hence a $(1+\epsilon)$-approximate solution) to $J$. As a result if $J$ is not $(k, \alpha)$-irreducible, then a $(1+\epsilon / 4)$-approximate solution to $J^{\prime}$ will bring us to a $(1+\epsilon)$-approximate solution to $J$. Hence everything boils down to the approximation of $(k, \alpha)$-irreducible instances.

\subsection{Extendable solutions}

By Lemma 5, if there is solution such that the sizes of all corresponding clusters are constant fractions of the number of the input vectors, then sampling produces a good approximate solution. However, there is no guarantee that such a favorable condition will occur. To overcome this we sample vectors for large clusters and then identify some vectors in the input which we can safely delete and make the next largest remaining cluster a constant fraction of the rest of the vectors. Towards that we need the following definition.

Definition 10 ( $\delta$-extension of a solution). Let $J=(X, k, \mathcal{R})$ be an instance of BINARY ConStrained Clustering and $\delta \geq 0$. Let $B \subseteq X$ and $C_{1} \subseteq\{0,1\}^{m}$ be a set of $k_{1}$ vectors for some $k_{1} \leq k$. The pair $\left(C_{1}, B\right)$ is called $\delta$-extendable for instance $J$ if there is a vector set $C_{2} \subseteq\{0,1\}^{m}$ of size $k-k_{1}$ such that $C_{1} \cup C_{2}$ satisfies $\mathcal{R}$ and $\operatorname{cost}\left(B, C_{1}\right)+\operatorname{cost}\left(X \backslash B, C_{1} \cup C_{2}\right) \leq(1+\delta) \mathrm{OPT}(J)$. We say that $C_{2}$ is a $\delta$-extension of $\left(C_{1}, B\right)$.

In particular, if $\left(C_{1}, B\right)$ is $\delta$-extendable for $J$, then there is a set $C \supseteq C_{1}$ such that $C$ is a solution to $J$ with cost at most $(1+\delta) \mathrm{OPT}(J)$ even when $B$ is assigned to the clusters corresponding to the center vectors in $C_{1}$. This implies that after we find such a set $C_{1}$, it is safe to delete the vectors $B$ from the input set of vectors. If $C_{2}$ is a $\delta$-extension of $\left(C_{1}, B\right)$, then there exist index subset $I \subseteq\{1, \ldots, k\}$ of size $\left|C_{1}\right|$ such that $C_{1}$ satisfies $\operatorname{proj}_{I}(\mathcal{R})$ and $C_{2}$ satisfies $\mathcal{R}\left(I, C_{1}\right)$. The proof of the next observation follows directly from the definition of $\delta$-extention.

Observation 6. Let $J=(X, k, \mathcal{R})$ be an instance of Binary Constrained Clustering, $\delta^{\prime} \geq \delta \geq 0$, and $\left(C_{1}, B\right)$ be a $\delta$-extendable pair for $J$. Then

- $\left(C_{1}, B\right)$ is also $\delta^{\prime}$-extendable for $J$,

- if $C_{2}$ is a $\delta$-extension of $\left(C_{1}, B\right)$, then $C_{2}$ is also a $\delta^{\prime}$-extension of $\left(C_{1}, B\right)$, and

- for each $B^{\prime} \subseteq B$, pair $\left(C_{1}, B^{\prime}\right)$ is $\delta$-extendable for $J$.

Let $\left(C_{1}, B\right)$ be a pair which is $\delta$-extendable for $J$ and $C \supseteq C_{1}$ be such that $C \backslash C_{1}$ is a $\delta$-extension of $\left(C_{1}, B\right)$. While the set $C \backslash C_{1}$ is not known to us and we do not know yet how to compute it, as we will see in the following lemma, we can proceed successfully even if only the 
minimum Hamming distance $t$ between vectors in $C_{1}$ and $C \backslash C_{1}$ is known. We show that if we know $t$, then we can find a set of vectors $B^{\prime} \subseteq X \backslash B$ such that $B^{\prime}$ is not only safe to delete but the number of vectors in $X \backslash\left(B \cup B^{\prime}\right)$ which will be assigned to clusters corresponding to $C_{1}$ (in the solution $C$ ) is at most a constant fraction of the number of vectors in $X \backslash\left(B \cup B^{\prime}\right)$. In other words the number of vectors which will be assigned to clusters corresponding to $C \backslash C_{1}$ will be at least a constant fraction of the number of vectors in $X \backslash\left(B \cup B^{\prime}\right)$. This information is of crucial importance; it yields that from the remaining set of vectors at least one of the clusters assigned to $C \backslash C_{1}$ is large, and thus could be found by sampling.

Lemma 7. Let $J=(X, k, \mathcal{R})$ be an instance of Binary Constrained Clustering and $\delta \geq 0$. Let $\left(C_{1}, B\right)$, where $B \subseteq X$ and $C_{1} \subseteq\{0,1\}^{m}$, be a $\delta$-extendable pair for $J$. Let $C_{2}$ be a $\delta$ extension of $\left(C_{1}, B\right)$ and $t=\min \left\{d_{H}\left(\mathbf{c}, \mathbf{c}^{\prime}\right): \mathbf{c} \in C_{1}, \mathbf{c}^{\prime} \in C_{2}\right\}$. Let $\left(Z_{1}, Z_{2}\right)$ be a partition of $X \backslash B$ such that $\operatorname{cost}\left(X \backslash B, C_{1} \cup C_{2}\right)=\operatorname{cost}\left(Z_{1}, C_{1}\right)+\operatorname{cost}\left(Z_{2}, C_{2}\right)$. Let $B^{\prime}=\bigcup_{\mathbf{c} \in C_{1}} \mathcal{B}(\mathbf{c}, t / 2) \cap(X \backslash B)$. Then the following conditions hold.

(i) $B^{\prime} \subseteq Z_{1}$. Moreover, $B^{\prime}$ consists of the first $\left|B^{\prime}\right|$ vectors of $X \backslash B$ in the ordering according to the non-decreasing distance $d_{H}\left(\mathbf{x}, C_{1}\right)$ (where $\left.\mathbf{x} \in X \backslash B\right)$.

(ii) $\operatorname{cost}\left(X \backslash\left(B \cup B^{\prime}\right), C_{1} \cup C_{2}\right)=\operatorname{cost}\left(Z_{1} \backslash B^{\prime}, C_{1}\right)+\operatorname{cost}\left(Z_{2}, C_{2}\right)$. Moreover, $\left(C_{1}, B \cup B^{\prime}\right)$ is $\delta$-extendable for $J$ and $C_{2}$ is a $\delta$-extension of $\left(C_{1}, B \cup B^{\prime}\right)$.

(iii) If $J$ is $\left(k, 5 \delta^{\prime}\right)$-irreducible for some $\delta^{\prime} \geq \delta$, then $\left|Z_{2}\right| \geq\left(\frac{\delta^{\prime}}{1+\delta^{\prime}}\right)\left|X \backslash\left(B \cup B^{\prime}\right)\right|$. If in addition, $\left|B^{\prime}\right| \leq \frac{|X \backslash B|}{2}$, then $\left|Z_{2}\right| \geq\left(\frac{\delta^{\prime}}{2\left(1+\delta^{\prime}\right)}\right)|X \backslash B|$.

Proof. We start with $(i)$. Since $t=\min \left\{d_{H}\left(\mathbf{c}, \mathbf{c}^{\prime}\right): \mathbf{c} \in C_{1}, \mathbf{c}^{\prime} \in C_{2}\right\}$, for any vector $\mathbf{x}$ in $\bigcup_{\mathbf{c} \in C_{1}} \mathcal{B}(\mathbf{c}, t / 2)$, the value $d_{H}\left(\mathbf{x}, C_{2}\right)$ is strictly greater than $t / 2$. Because $\operatorname{cost}\left(X \backslash B, C_{1} \cup\right.$ $\left.C_{2}\right)=\operatorname{cost}\left(Z_{1}, C_{1}\right)+\operatorname{cost}\left(Z_{2}, C_{2}\right)$, we conclude that $B^{\prime} \subseteq Z_{1}$. Now if we order the vectors of $X \backslash B$ according to their distances to $C_{1}$ (the smallest distance comes first and ties are broken arbitrarily), then the first $\left|B^{\prime}\right|$ vectors in this ordering are within distance strictly less than $t / 2$ from $C_{1}$, while for any other vector $\mathbf{x} \notin B^{\prime}, d_{H}\left(\mathbf{x}, C_{1}\right) \geq t / 2$. This concludes the proof of $(i)$.

Before proceeding with $(i i)$ and (iii), we observe the following. First, since $C_{2}$ is a $\delta$-extension of $\left(C_{1}, B\right)$, we have that

$$
\operatorname{cost}\left(X, C_{1} \cup C_{2}\right) \leq \operatorname{cost}\left(B, C_{1}\right)+\operatorname{cost}\left(X \backslash B, C_{1} \cup C_{2}\right) \leq(1+\delta) \mathrm{OPT}(J) .
$$

By the definition of sets $Z_{1}$ and $Z_{2}$, we have that

$$
\begin{aligned}
\operatorname{cost}\left(B, C_{1}\right)+\operatorname{cost}\left(X \backslash B, C_{1} \cup C_{2}\right) & =\operatorname{cost}\left(B, C_{1}\right)+\operatorname{cost}\left(Z_{1}, C_{1}\right)+\operatorname{cost}\left(Z_{2}, C_{2}\right) \\
& =\operatorname{cost}\left(B \cup Z_{1}, C_{1}\right)+\operatorname{cost}\left(Z_{2}, C_{2}\right) .
\end{aligned}
$$

Thus, $\quad \operatorname{cost}\left(X, C_{1} \cup C_{2}\right) \leq \operatorname{cost}\left(B \cup Z_{1}, C_{1}\right)+\operatorname{cost}\left(Z_{2}, C_{2}\right) \leq(1+\delta) \mathrm{OPT}(J)$.

We need one more observation and its proof follows directly from the definition of sets $Z_{1}$ and $Z_{2}$.

Observation 7. For every $\mathbf{x} \in Z_{1}, d_{H}\left(\mathbf{x}, C_{1} \cup C_{2}\right)=d_{H}\left(\mathbf{x}, C_{1}\right)$ and for every $\mathbf{y} \in Z_{2}, d_{H}\left(\mathbf{y}, C_{1} \cup\right.$ $\left.C_{2}\right)=d_{H}\left(\mathbf{y}, C_{2}\right)$.

Now we prove condition (ii). First, by Observation 7 and the fact that $B^{\prime} \subseteq Z_{1}$, we have that

$$
\operatorname{cost}\left(X \backslash\left(B \cup B^{\prime}\right), C_{1} \cup C_{2}\right)=\operatorname{cost}\left(Z_{1} \backslash B^{\prime}, C_{1}\right)+\operatorname{cost}\left(Z_{2}, C_{2}\right) .
$$


To prove that $\left(C_{1}, B \cup B^{\prime}\right)$ is $\delta$-extendable for $J$ and that $C_{2}$ is a $\delta$-extension of $\left(C_{1}, B \cup B^{\prime}\right)$, we note that

$$
\begin{aligned}
\operatorname{cost}\left(B \cup B^{\prime}, C_{1}\right) & +\operatorname{cost}\left(X \backslash\left(B \cup B^{\prime}\right), C_{1} \cup C_{2}\right) \\
& =\operatorname{cost}\left(B \cup B^{\prime}, C_{1}\right)+\operatorname{cost}\left(Z_{1} \backslash B^{\prime}, C_{1}\right)+\operatorname{cost}\left(Z_{2}, C_{2}\right) \quad \text { (by (24)) } \\
& \left.\leq(1+\delta) \mathrm{OPT}(J) . \quad \text { (by (23) and because } B^{\prime} \subseteq Z_{1}\right)
\end{aligned}
$$

Now we prove condition (iii) of the lemma. Let $C_{2}=\left\{\mathbf{c}_{1}, \ldots, \mathbf{c}_{\ell}\right\}$, where $\ell=k-\left|C_{1}\right|$. Let $Y_{1} \uplus \cdots \uplus Y_{\ell}$ be a partition of $Z_{2}$ such that

$$
\operatorname{cost}\left(Z_{2}, C_{2}\right)=\sum_{j=1}^{\ell} \sum_{\mathbf{y} \in Y_{j}} d_{H}\left(\mathbf{c}_{j}, \mathbf{y}\right) .
$$

Let vector $\mathbf{c} \in C_{1}$ and index $r \leq \ell$ be such that $t=d_{H}\left(\mathbf{c}, \mathbf{c}_{r}\right)$.

Claim 4. If $J$ is $\left(k, 5 \delta^{\prime}\right)$-irreducible for some $\delta^{\prime} \geq \delta$, then $\left|Z_{1} \backslash B^{\prime}\right| \leq \frac{1}{\delta^{\prime}} \cdot\left|Y_{r}\right|$.

Proof. For the sake of contradiction assume that $\left|Z_{1} \backslash B^{\prime}\right|>\frac{1}{\delta^{\prime}} \cdot\left|Y_{r}\right|$. This implies that $\operatorname{cost}\left(Z_{1} \backslash\right.$ $\left.B^{\prime}, C_{1}\right) \geq \frac{t}{2 \delta^{\prime}}\left|Y_{r}\right|$. Thus

$$
t\left|Y_{r}\right| \leq 2 \delta^{\prime} \operatorname{cost}\left(Z_{1} \backslash B^{\prime}, C_{1}\right) \leq 2 \delta^{\prime} \operatorname{cost}\left(Z_{1}, C_{1}\right) .
$$

Now we upper bound $\operatorname{cost}\left(X, C_{1} \cup\left(C_{2} \backslash\left\{c_{r}\right\}\right)\right)-\operatorname{cost}\left(X, C_{1} \cup C_{2}\right)$, by considering the the change in cost when we reassign the vectors in $Y_{r}$ to the cluster with center c.

$$
\begin{aligned}
\operatorname{cost}\left(X, C_{1} \cup\left(C_{2} \backslash\left\{\mathbf{c}_{r}\right\}\right)\right) & -\operatorname{cost}\left(X, C_{1} \cup C_{2}\right) \\
& \leq \sum_{\mathbf{y} \in Y_{r}}\left(d_{H}(\mathbf{y}, \mathbf{c})-d_{H}\left(\mathbf{y}, \mathbf{c}_{r}\right)\right) \\
& \leq \sum_{\mathbf{y} \in Y_{r}} d_{H}\left(\mathbf{c}, \mathbf{c}_{r}\right) \quad \text { (by the triangle inequality) } \\
& =\left|Y_{r}\right| \cdot t \leq 2 \delta^{\prime} \cdot \operatorname{cost}\left(Z_{1}, C_{1}\right)
\end{aligned}
$$

But then

$$
\begin{aligned}
\mathrm{OPT}_{k-1}(J) & \leq \operatorname{cost}\left(X, C_{1} \cup\left(C_{2} \backslash\left\{\mathbf{c}_{r}\right\}\right)\right) \\
& \leq \operatorname{cost}\left(X, C_{1} \cup C_{2}\right)+2 \delta^{\prime} \operatorname{cost}\left(Z_{1}, C_{1}\right) \quad(\text { by }(26)) \\
& \leq(1+\delta) \cdot \mathrm{OPT}(J)+2 \delta^{\prime}(1+\delta) \cdot \mathrm{OPT}(J) \quad(\text { by }(23)) \\
& \leq\left(1+\delta+4 \delta^{\prime}\right) \cdot \mathrm{OPT}(J) \quad(\text { since } \delta \leq 1) \\
& \leq\left(1+5 \delta^{\prime}\right) \cdot \mathrm{OPT}(J) . \quad\left(\text { since } \delta \leq \delta^{\prime}\right)
\end{aligned}
$$

This contradicts the assumption that $J$ is $\left(k, 5 \delta^{\prime}\right)$-irreducible.

Since $Y_{r} \subseteq Z_{2}$, by Claim 4 , we have that $\left|Z_{1} \backslash B^{\prime}\right| \leq \frac{1}{\delta^{\prime}} \cdot\left|Z_{2}\right|$. Sets $Z_{1}$ and $Z_{2}$ form a partition of $X \backslash B$ and because $B^{\prime} \subseteq Z_{1}$, we have that $X \backslash\left(B \cup B^{\prime}\right)=\left(Z_{1} \backslash B^{\prime}\right) \cup Z_{2}$. This implies that $\left|X \backslash\left(B \cup B^{\prime}\right)\right| \leq\left|Z_{2}\right|\left(1+1 / \delta^{\prime}\right)$, and hence

$$
\left|Z_{2}\right| \geq \frac{\delta^{\prime}}{1+\delta^{\prime}} \cdot\left|X \backslash\left(B \cup B^{\prime}\right)\right| .
$$


Finally, we prove the last condition in (iii). If $\left|B^{\prime}\right| \leq \frac{|X \backslash B|}{2}$, then

$$
\begin{aligned}
\left|Z_{2}\right| & \geq \frac{\delta^{\prime}}{1+\delta^{\prime}} \cdot\left|X \backslash\left(B \cup B^{\prime}\right)\right| \\
& \geq \frac{\delta^{\prime}}{1+\delta^{\prime}} \cdot\left(|X \backslash B|-\left|B^{\prime}\right|\right) \\
& \geq \frac{\delta^{\prime}}{2\left(1+\delta^{\prime}\right)} \cdot|X \backslash B| .
\end{aligned}
$$

This completes the proof of the lemma.

Due to Lemma 7 , once we have a partial solution $C_{1}$, we can identify a set $B^{\prime}$ of vectors such that the number of vectors in the largest cluster corresponding to $C \backslash C_{1}$ (here $C$ is a good solution which contains $C_{1}$ ) is at least a constant fraction of the remaining vectors. This allows us to further use Lemma 5 as formally explained in Lemma 9. To make the statements in Lemma 9 easier, we make use of one of the best $\delta$-extension of $\left(C_{1}, B\right)$ as derived in the following lemma.

Definition 11 (Good $\delta$-extension). For instance $J=\left(X, k, \mathcal{R}=\left\{R_{1}, \ldots, R_{m}\right\}\right)$ of BinaRY Constrained Clustering, $\delta \geq 0, B \subseteq X$ and $C_{1} \subseteq\{0,1\}^{m}$ of size $k^{\prime}$, a $\delta$-extension $C_{2}$ of $\left(C_{1}, B\right)$ is a good $\delta$-extension of $\left(C_{1}, B\right)$ if there is a partition $Z_{1} \uplus Z_{2}=X \backslash B$ and index set $I \subseteq\{1, \ldots, k\}$ of size $k^{\prime}$ such that

- $\operatorname{cost}\left(X \backslash B, C_{1} \cup C_{2}\right)=\operatorname{cost}\left(Z_{1}, C_{1}\right)+\operatorname{cost}\left(Z_{2}, C_{2}\right)$,

- $C_{1}$ satisfies $\operatorname{proj}_{I}(\mathcal{R})$, and

- $C_{2}$ is an optimal solution to $J^{\prime}=\left(Z_{2}, k-k^{\prime}, \mathcal{R}^{\prime}=\mathcal{R}\left(I, C_{1}\right)\right)$.

We will refer to $J^{\prime}$ as to the $C_{2}$-optimal reduced instance.

Lemma 8. Let $J=\left(X, k, \mathcal{R}=\left\{R_{1}, \ldots, R_{m}\right\}\right)$ be an instance of Binary Constrained ClusTERING and $\delta \geq 0$. Let $B \subseteq X$ and $C_{1} \subseteq\{0,1\}^{m}$ be a set of $k^{\prime}$ vectors for some $k^{\prime} \leq k$ such that $\left(C_{1}, B\right)$ is $\delta$-extendable for $J$. Then there is a good $\delta$-extension $C_{2}$ of $\left(C_{1}, B\right)$.

Proof. Among all $\delta$-extensions of $\left(C_{1}, B\right)$, let $C_{2}$ be a $\delta$-extension of $\left(C_{1}, B\right)$ such that $\operatorname{cost}(X \backslash$ $\left.B, C_{1} \cup C_{2}\right)$ is minimized. We prove that $C_{2}$ is the required $\delta$-extension of $\left(C_{1}, B\right)$.

Let

$$
\eta=\operatorname{cost}\left(X \backslash B, C_{1} \cup C_{2}\right) .
$$

Since $C_{2}$ is a $\delta$-extension of $\left(C_{1}, B\right)$, there is a set $I \subseteq\{1, \ldots, k\}$ of size $k^{\prime}$ and a partition $Z_{1} \uplus Z_{2}$ of $X \backslash B$ such that $C_{1}$ and $C_{2}$ satisfy families of relations $\operatorname{proj}_{I}(\mathcal{R})$ and $\mathcal{R}\left(I, C_{1}\right)$ correspondingly, and

$$
\eta=\operatorname{cost}\left(Z_{1}, C_{1}\right)+\operatorname{cost}\left(Z_{2}, C_{2}\right) \leq(1+\delta) \mathrm{OPT}(J) .
$$

By the choice of $C_{2}$, for every $\delta$-extension $C_{2}^{\prime}$ of $\left(C_{1}, B\right)$, we have that $\operatorname{cost}\left(X \backslash B, C_{1} \cup C_{2}^{\prime}\right) \geq \eta$. Assume, for the sake of contradiction, that $C_{2}$ is not an optimal solution to $\left(Z_{2}, k-k^{\prime}, \mathcal{R}\left(I, C_{1}\right)\right)$. Let $C_{2}^{*}$ be an optimal solution to $\left(Z_{2}, k-k^{\prime}, \mathcal{R}\left(I, C_{1}\right)\right)$. Then $\operatorname{cost}\left(Z_{2}, C_{2}^{*}\right)<\operatorname{cost}\left(Z_{2}, C_{2}\right)$. Moreover, $C_{1} \cup C_{2}^{*}$ satisfies $\mathcal{R}$ and hence is a solution to $J$. Since $\operatorname{cost}\left(Z_{2}, C_{2}^{*}\right)<\operatorname{cost}\left(Z_{2}, C_{2}\right)$, we conclude that

$$
\operatorname{cost}\left(X \backslash B, C_{1} \cup C_{2}^{*}\right) \leq \operatorname{cost}\left(Z_{1}, C_{1}\right)+\operatorname{cost}\left(Z_{2}, C_{2}^{*}\right)<\operatorname{cost}\left(Z_{1}, C_{1}\right)+\operatorname{cost}\left(Z_{2}, C_{2}\right)=\eta .
$$

This contradicts the choice of $C_{2}$, which in turn, completes the proof of the lemma. 
In order to state the next lemma, we need one more definition.

Definition 12 (Set of $\kappa$-heavy clusters). Let $\kappa>0, X$ be a vector set and $X_{1}, \ldots, X_{\ell}$ be a clustering of $X$. We say that a subset of clusters $\mathcal{X} \subseteq\left\{X_{1}, \ldots, X_{\ell}\right\}$ is the set of $\kappa$-heavy clusters if for every $Y \in\{\mathcal{X}\}$ and $Z \notin \mathcal{X},|Y|>\kappa|Z|$.

The following lemma says that if we have an irreducible instance $J$ with $\delta$-extendable pair $\left(B, C_{1}\right)$, then it is possible to construct a larger extendable pair by adding to $C_{1}$ a set of "approximate" centers of heavy clusters from optimal clustering of instance $J^{\prime}$ obtained from $J$ by "subtracting" $\left(C_{1}, B\right)$.

Lemma 9. Let $\delta \geq 0$ and $0 \leq \alpha \leq 1$ and let $J=\left(X, k, \mathcal{R}=\left\{R_{1}, \ldots, R_{m}\right\}\right)$ be a $\left(k, 5 \delta^{\prime}\right)$ irreducible instance of Binary Constrained Clustering for some $\delta^{\prime} \geq \delta$. Let pair $\left(C_{1}, B\right)$, $B \subseteq X, C_{1} \subseteq\{0,1\}^{m},\left|C_{1}\right|<k$, be $\delta$-extendable for $J$. Let $C_{2}=\left\{\mathbf{c}_{1}^{*}, \ldots, \mathbf{c}_{\ell}^{*}\right\}$ be a good $\delta$ extension of $\left(C_{1}, B\right)$ and $J^{\prime}=\left(Z_{2}, \ell=k-\left|C_{1}\right|, \mathcal{R}^{\prime}=\mathcal{R}\left(I, C_{1}\right)\right)$ be the corresponding $C_{2}$-optimal reduced instance. (The existence of such $C_{2}$ is guaranteed by Lemma 8.) Let also $X_{1}, \ldots, X_{\ell}$ be the set of clusters corresponding to the solution $C_{2}$ of $J^{\prime}$ and let $I^{\prime} \subseteq\{1, \ldots, \ell\},\left|I^{\prime}\right|=k^{\prime}$, be the set of indices of the $\frac{k}{\alpha}$-heavy clusters from this set.

Then for every solution $C_{2}^{\prime}=\left\{\mathbf{c}_{i}: i \in I^{\prime}\right\}$ to $J_{1}^{\prime}=\left(\bigcup_{i \in I^{\prime}} X_{i}, k^{\prime}, \operatorname{proj}_{I^{\prime}}\left(\mathcal{R}^{\prime}\right)\right)$ satisfying condition

$$
\sum_{j \in I^{\prime}} \operatorname{cost}\left(X_{j},\left\{\mathbf{c}_{j}\right\}\right) \leq(1+\alpha) \cdot \sum_{j \in I^{\prime}} \operatorname{cost}\left(X_{j},\left\{\mathbf{c}_{j}^{*}\right\}\right)
$$

the pair $\left(C_{1} \cup C_{2}^{\prime}, B\right)$ is $(5 \delta+4 \alpha)$-extendable for $J$.

Proof. Because $C_{2}$ is a $\delta$-extension of $\left(C_{1}, B\right)$, we have that

$$
\operatorname{cost}\left(B, C_{1}\right)+\operatorname{cost}\left(X \backslash B, C_{1} \cup C_{2}\right) \leq(1+\delta) \mathrm{OPT}(J) .
$$

The assumption that $C_{2}$ is a good $\delta$-extension of $\left(C_{1}, B\right)$, yields that there are $Z_{1} \uplus Z_{2}=X \backslash B$ and $I \in\left(\begin{array}{c}{[k]} \\ \left|C_{1}\right|\end{array}\right)$ such that

- $\operatorname{cost}\left(X \backslash B, C_{1} \cup C_{2}\right)=\operatorname{cost}\left(Z_{1}, C_{1}\right)+\operatorname{cost}\left(Z_{2}, C_{2}\right)$,

- $C_{1}$ satisfies $\operatorname{proj}_{I}(\mathcal{R})$, and

- $C_{2}$ is an optimal solution to $J^{\prime}=\left(Z_{2}, \ell=k-\left|C_{1}\right|, \mathcal{R}^{\prime}=\mathcal{R}\left(I, C_{1}\right)\right)$.

Hence

$$
\operatorname{cost}\left(X, C_{1} \cup C_{2}\right) \leq \operatorname{cost}\left(B \cup Z_{1}, C_{1}\right)+\operatorname{cost}\left(Z_{2}, C_{2}\right) \leq(1+\delta) \mathrm{OPT}(J) .
$$

For the ease of presentation we assume that $I^{\prime}=\left\{1, \ldots, k^{\prime}\right\}$. Let $W_{1}=\bigcup_{j=I^{\prime}} X_{j}$ and $W_{2}=Z_{2} \backslash W_{1}$. Let $C_{3}=\left\{\mathbf{c}_{k^{\prime}+1}, \ldots, \mathbf{c}_{\ell}\right\}$ be an optimal solution to $J_{2}^{\prime}=\left(W_{2}, \ell-k^{\prime}, \mathcal{R}^{\prime}\left(I^{\prime}, C_{2}^{\prime}\right)\right)$. From the solution $C_{3}$ of $J_{2}^{\prime}$, we define a solution $C_{3}^{\prime}=\left\{\mathbf{c}_{k^{\prime}+1}^{\prime}, \ldots, \mathbf{c}_{\ell}^{\prime}\right\}$ to $J_{2}^{\prime}$ as follows. For each $i \in[m]$, we set

$$
\left(\mathbf{c}_{k^{\prime}+1}^{\prime}[i], \ldots, \mathbf{c}_{\ell}^{\prime}[i]\right)= \begin{cases}\left(\mathbf{c}_{k^{\prime}+1}^{*}[i], \ldots, \mathbf{c}_{\ell}^{*}[i]\right), & \text { if }\left(\mathbf{c}_{1}[i], \ldots, \mathbf{c}_{k^{\prime}}[i]\right)=\left(\mathbf{c}_{1}^{*}[i], \ldots, \mathbf{c}_{k^{\prime}}^{*}[i]\right), \\ \left(\mathbf{c}_{k^{\prime}+1}[i], \ldots, \mathbf{c}_{\ell}[i]\right), & \text { otherwise. }\end{cases}
$$

Observation 8. $C_{3}^{\prime}$ is a solution to $J_{2}^{\prime}$.

Proof. From the definition of $C_{3}^{\prime}$, we have that for any $i \in[m],\left(\mathbf{c}_{k^{\prime}+1}^{\prime}[i], \ldots, \mathbf{c}_{\ell}^{\prime}[i]\right) \in \mathcal{R}^{\prime}\left(I^{\prime}, C_{2}^{\prime}\right)$. Hence $C_{3}^{\prime}$ is a solution to $J_{2}$. 
Let us remind that we define $\mathcal{R}^{\prime}=\mathcal{R}\left(I, C_{1}\right)$. Because $C_{1} \in \operatorname{proj}_{I}(\mathcal{R}), C_{2}^{\prime} \in \operatorname{proj}_{I^{\prime}}\left(\mathcal{R}^{\prime}\right)$ and $C_{3}^{\prime} \in \mathcal{R}^{\prime}\left(I^{\prime}, C_{2}^{\prime}\right)$, we have that $C_{1} \cup C_{2}^{\prime} \cup C_{3}^{\prime}$ is a solution to $J$. We will prove that $C_{3}^{\prime}$ is indeed a $(5 \delta+4 \alpha)$-extension of $C_{1} \cup C_{2}^{\prime}$. Towards that we define

$$
\Delta_{1}=\sum_{j \in I^{\prime}} \sum_{\mathbf{x} \in X_{j}} d_{H}\left(\mathbf{x}, \mathbf{c}_{j}^{*}\right)=\sum_{j \in I^{\prime}} \operatorname{cost}\left(X_{j},\left\{\mathbf{c}_{j}^{*}\right\}\right)
$$

and

$$
\Delta_{2}=\sum_{j \in[\ell] \backslash I^{\prime}} \sum_{\mathbf{x} \in X_{j}} d_{H}\left(\mathbf{x}, \mathbf{c}_{j}^{*}\right)=\sum_{j \in[\ell] \backslash I^{\prime}} \operatorname{cost}\left(X_{j},\left\{\mathbf{c}_{j}^{*}\right\}\right)
$$

By counting the number of mismatches at each of the coordinates of vectors in $W_{1}$ with its corresponding center in $\left\{\mathbf{c}_{1}^{*}, \ldots, \mathbf{c}_{k^{\prime}}^{*}\right\}$, we get that

$$
\sum_{r=1}^{m} \sum_{j \in I^{\prime}} \sum_{\mathbf{x} \in X_{j}: \mathbf{x}[r] \neq \mathbf{c}_{j}^{*}[r]} 1=\sum_{j \in I^{\prime}} \operatorname{cost}\left(X_{j},\left\{\mathbf{c}_{j}^{*}\right\}\right)=\Delta_{1},
$$

where the last equality follows from (28). Since $C$ is an optimal solution to $J^{\prime}$ with corresponding clusters $X_{1}, \ldots, X_{\ell}$, we have that OPT $\left(J^{\prime}\right)=\Delta_{1}+\Delta_{2}$. Combining (28) with the assumption $\sum_{j \in I^{\prime}} \operatorname{cost}\left(X_{j},\left\{\mathbf{c}_{j}\right\}\right) \leq(1+\alpha)\left(\sum_{j \in I^{\prime}} \operatorname{cost}\left(X_{j},\left\{\mathbf{c}_{j}^{*}\right\}\right)\right)$, we have that

$$
\operatorname{cost}\left(W_{1}, C_{2}^{\prime}\right) \leq \sum_{j \in I^{\prime}} \operatorname{cost}\left(X_{j},\left\{\mathbf{c}_{j}\right\}\right) \leq(1+\alpha) \Delta_{1} .
$$

By arguments similar to the reasoning for (30) and by (31), we obtain the following.

$$
\sum_{r=1}^{m} \sum_{j \in I^{\prime}} \sum_{\mathbf{x} \in X_{j}: \mathbf{x}[r] \neq \mathbf{c}_{j}[r]} 1=\sum_{j \in I^{\prime}} \operatorname{cost}\left(X_{j},\left\{\mathbf{c}_{j}\right\}\right)=(1+\alpha) \Delta_{1} .
$$

We claim that

Claim 5. $\operatorname{cost}\left(W_{2}, C_{3}^{\prime}\right) \leq \Delta_{2}+\left(3 \alpha \cdot \Delta_{1}\right)$

Before proving the claim, let us show how it concludes the proof of the lemma. By (31) and Claim 5, we have that

$$
\begin{aligned}
\operatorname{cost}\left(W_{1}, C_{2}^{\prime}\right)+\operatorname{cost}\left(W_{2}, C_{3}^{\prime}\right) & \leq(1+\alpha) \Delta_{1}+\Delta_{2}+\left(3 \alpha \cdot \Delta_{1}\right) \\
& \leq(1+4 \alpha)\left(\Delta_{1}+\Delta_{2}\right) \\
& \leq(1+4 \alpha) \operatorname{cost}\left(Z_{2}, C_{2}\right)
\end{aligned}
$$

where the last inequality follows from the fact that $C_{2}=\left\{\mathbf{c}_{1}^{*}, \ldots, \mathbf{c}_{\ell}^{*}\right\}$ is a solution to $J^{\prime}$ with corresponding clusters $X_{1}, \ldots, X_{\ell}$ and equations (28) and (29).

Now we prove that $C_{3}^{\prime}$ is a $(5 \delta+4 \alpha)$-extension of $\left(C_{1} \cup C_{2}^{\prime}, B\right)$ for $J$. Towards that we upper bound $\eta=\operatorname{cost}\left(B, C_{1} \cup C_{2}^{\prime}\right)+\operatorname{cost}\left(X \backslash B, C_{1} \cup C_{2}^{\prime} \cup C_{3}^{\prime}\right)$.

$$
\begin{array}{rlr}
\eta & \leq \operatorname{cost}\left(B, C_{1}\right)+\operatorname{cost}\left(Z_{1}, C_{1}\right)+\operatorname{cost}\left(W_{1}, C_{2}^{\prime}\right)+\operatorname{cost}\left(W_{2}, C_{3}^{\prime}\right) \\
& \leq \operatorname{cost}\left(B, C_{1}\right)+\operatorname{cost}\left(Z_{1}, C_{1}\right)+(1+4 \alpha) \operatorname{cost}\left(Z_{2}, C_{2}\right) & (\text { by } \\
& \leq(1+\delta) \mathrm{OPT}(J)+(4 \alpha) \operatorname{cost}\left(Z_{2}, C_{2}\right) & (\text { by }(27)) \\
& \leq(1+\delta) \mathrm{OPT}(J)+(4 \alpha)(1+\delta) \mathrm{OPT}(J) & (\text { by }(27)) \\
& \leq(1+5 \delta+4 \alpha) \mathrm{OPT}(J)
\end{array}
$$

This, subject to the proof of Claim 5, completes the proof of the lemma. 
Proof of Claim 5. We need one more technical claim.

Claim 6. For any $j \in[\ell] \backslash I^{\prime}$,

$$
\sum_{r \in[m]: \mathbf{c}_{j}^{\prime}[r] \neq \mathbf{c}_{j}^{*}[r]}\left|X_{j}\right| \leq \frac{3 \alpha}{k} \cdot \Delta_{1} .
$$

Proof. Fix $j \in[\ell] \backslash I^{\prime}$. For any $r \in[m]$ such that $\mathbf{c}_{j}^{\prime}[r] \neq \mathbf{c}_{j}^{*}[r]$, by the definition of $\left(\mathbf{c}_{k^{\prime}+1}^{\prime}[r], \ldots, \mathbf{c}_{k}^{\prime}[r]\right)$, we have that $\left(\mathbf{c}_{1}[r], \ldots, \mathbf{c}_{k^{\prime}}[r]\right) \neq\left(\mathbf{c}_{1}^{*}[r], \ldots, \mathbf{c}_{k^{\prime}}^{*}[r]\right)$. That is, for any $r \in[m]$ such that $\mathbf{c}_{j}^{\prime}[r] \neq$ $\mathbf{c}_{j}^{*}[r]$, there is $j_{r} \in I^{\prime}$ such that $\mathbf{c}_{j_{r}}[r] \neq \mathbf{c}_{j_{r}}^{*}[r]$. Define a map $f$ from $I_{j}=\left\{r \in[m]: \mathbf{c}_{j}^{\prime}[r] \neq \mathbf{c}_{j}^{*}[r]\right\}$ to $I^{\prime}$ as follows: $f(r)=j_{r}$, where $j_{r}$ is an arbitrary index in $I^{\prime}$ such that $\mathbf{c}_{j_{r}}[r] \neq \mathbf{c}_{j_{r}}^{*}[r]$. We define sets

$$
A[r]=\left\{\mathbf{x} \in X_{f(r)} \mid \mathbf{x}[r] \neq \mathbf{c}_{f(r)}[r]\right\},
$$

and

$$
B[r]=\left\{\mathbf{x} \in X_{f(r)} \mid \mathbf{x}[r] \neq \mathbf{c}_{f(r)}^{*}[r]\right\} .
$$

Since $\left|X_{j}\right|<\frac{\alpha}{k}\left|X_{f(r)}\right|$ for every $r \in I_{j}$ (by the assumption that clusters with indices from $I^{\prime}$ are $\frac{k}{\alpha}$-heavy), we have that

$$
\left|X_{j}\right| \leq \frac{\alpha}{k}\left|X_{f(r)}\right|=\frac{\alpha}{k}\left(\sum_{\mathbf{x} \in A[r]} 1+\sum_{\mathbf{x} \in B[r]} 1\right) .
$$

Therefore,

$$
\begin{aligned}
& \sum_{r \in[m]: \mathbf{c}_{j}^{\prime}[r] \neq \mathbf{c}_{j}^{*}[r]}\left|X_{j}\right| \leq \sum_{r \in[m]: \mathbf{c}_{j}^{\prime}[r] \neq \mathbf{c}_{j}^{*}[r]} \frac{\alpha}{k}\left(\sum_{\mathbf{x} \in A[r]} 1+\sum_{\mathbf{x} \in B[r]} 1\right) \\
\leq & \frac{\alpha}{k}\left(\left(\sum_{r \in[m]: \mathbf{c}_{j}^{\prime}[r] \neq \mathbf{c}_{j}^{*}[r]} \sum_{\mathbf{x} \in A[r]} 1\right)+\left(\sum_{r \in[m]: \mathbf{c}_{j}^{\prime}[r] \neq \mathbf{c}_{j}^{*}[r]} \sum_{\mathbf{x} \in B[r]} 1\right)\right) \\
\leq & \frac{\alpha}{k}\left(\left(\sum_{r \in[m]: \mathbf{c}_{j}^{\prime}[r] \neq \mathbf{c}_{j}^{*}[r]} \sum_{\mathbf{x} \in A[r]} 1\right)+\Delta_{1}\right) \quad(\text { by }(30)) \\
\leq & \frac{\alpha}{k}\left((1+\alpha) \Delta_{1}+\Delta_{1}\right) \quad(\text { by }(32)) \\
\leq & \frac{3 \alpha}{k} \Delta_{1} . \quad(\text { since } \alpha \leq 1)
\end{aligned}
$$

Now we are ready to proceed with the proof of Claim 5 . We bound 


$$
\begin{aligned}
\operatorname{cost}\left(W_{2}, C_{3}^{\prime}\right) & \leq \sum_{j \in[\ell] \backslash I^{\prime}} \sum_{\mathbf{x} \in X_{j}} d_{H}\left(\mathbf{x}, \mathbf{c}_{j}^{\prime}\right) \\
& =\sum_{j \in[\ell] \backslash I^{\prime}} \sum_{\mathbf{x} \in X_{j}} \sum_{r=1}^{m}\left|\mathbf{x}[r]-\mathbf{c}_{j}^{\prime}[r]\right| \\
& =\sum_{j \in[\ell] \backslash I^{\prime}} \sum_{\mathbf{x} \in X_{j}}\left(\sum_{r \in[m]: \mathbf{c}_{j}^{\prime}[r]=\mathbf{c}_{j}^{*}[r]}\left|\mathbf{x}[r]-\mathbf{c}_{j}^{\prime}[r]\right|+\sum_{r \in[m]: \mathbf{c}_{j}^{\prime}[r] \neq \mathbf{c}_{j}^{*}[r]}\left|\mathbf{x}[r]-\mathbf{c}_{j}^{\prime}[r]\right|\right) \\
& \leq \sum_{j \in[\ell] \backslash I^{\prime}} \sum_{\mathbf{x} \in X_{j}}\left(d_{H}\left(\mathbf{x}, \mathbf{c}_{j}^{*}\right)+\sum_{r \in[m]: \mathbf{c}_{j}^{\prime}[r] \neq \mathbf{c}_{j}^{*}[r]}\left|\mathbf{x}[r]-\mathbf{c}_{j}^{\prime}[r]\right|\right) \\
& =\Delta_{2}+\sum_{j \in[\ell] \backslash I^{\prime}} \sum_{\mathbf{x} \in X_{j}} \sum_{r \in[m]: \mathbf{c}_{j}^{\prime}[r] \neq \mathbf{c}_{j}^{*}[r]}\left|\mathbf{x}[r]-\mathbf{c}_{j}^{\prime}[r]\right| \quad(\text { by }(29)) \\
& \leq \Delta_{2}+\sum_{j \in[\ell] \backslash I^{\prime}} \sum_{r \in[m]: \mathbf{c}_{j}^{\prime}[r] \neq \mathbf{c}_{j}^{*}[r] \mathbf{x} \in X_{j}}\left|\mathbf{x}[r]-\mathbf{c}_{j}^{\prime}[r]\right| \\
& \leq \Delta_{2}+\sum_{j \in[\ell] \backslash I^{\prime}}\left|\sum_{r \in[m]: \mathbf{c}_{j}^{\prime}[r] \neq \mathbf{c}_{j}^{*}[r]}\right| X_{j} \mid \\
& \leq \Delta_{2}+\sum_{j \in[\ell] \backslash I^{\prime}} \frac{3 \alpha}{k} \cdot \Delta_{1} \quad \text { (by Claim 6) }
\end{aligned}
$$

The last inequality completes the proof of Claim 5, which in turn completes the proof of the lemma.

\section{Putting all together: Proof of Theorem 2}

As we already mentioned in Section 7, the most essential part of the proof of Theorem 2 is to approximate irreducible instances. We start from the approximation algorithm for irreducible instances and then use this algorithm to prove Theorem 2.

\subsection{Approximating irreducible instances}

Now we have sufficient ingredients to design an algorithm for outputting a $(1+\varepsilon)$-approximate solution under the assumption that the given instance is $\left(k, \frac{\varepsilon}{8 k^{*}}\right)$-irreducible for some $k^{*} \geq k$. More precisely, in this section we prove the following theorem.

Theorem 9. There is an algorithm with the following specifications.

- The input of the algorithm is an instance $J=\left(X, k, \mathcal{R}=\left\{R_{1}, \ldots, R_{m}\right\}\right)$ of BInARY Constrained Clustering, $\varepsilon>0$, and $k^{*} \geq k$ such that $J$ is $\left(k, \frac{\varepsilon}{8 k^{*}}\right)$-irreducible.

- The output of the algorithm is a solution $C$ to $J$ such that $\operatorname{cost}(X, C) \leq\left(1+\frac{\varepsilon}{40 k^{*}}\right) \mathrm{OPT}(J)$ with probability at least

$$
p\left(k^{*}, k, \varepsilon\right)=\left(\frac{\varepsilon}{1+\varepsilon}\right)^{k} \cdot\left(\frac{\varepsilon^{k}}{\left(40 k \cdot k^{*} \cdot\left(5^{k}-1\right)\right)^{k-1} \cdot 2 k \cdot\left(40 k^{*}+\varepsilon\right)}\right)^{\frac{c^{\prime} \cdot k^{2}}{\varepsilon^{2}} \log \frac{1}{\varepsilon}} .
$$


- The running time of the algorithm is $2^{\mathcal{O}\left(k^{2}+k \log \left(k^{*}\right)\right)} \cdot n \cdot m \cdot\left(\frac{1}{\varepsilon}\right)^{\mathcal{O}(k)}$.

First we provide an overview of the algorithm informally without mentioning actual values of the parameters. Then we reason about how to set different parameters which will lead to the required algorithm. And then we give a formal proof of Theorem 9 .

On a high level our algorithm works as follows. Let $J=(X, k, \mathcal{R})$ be the input instance. We consider a tuple $\left(C^{\prime}, S, \delta\right)$ to be a partial solution where $S \subseteq X$ and $C^{\prime} \subseteq\{0,1\}^{m},\left|C^{\prime}\right| \leq k$ and $\delta \geq 0$. We say that a partial solution $\left(C^{\prime}, S, \delta\right)$ is a good partial solution, if $\left(C^{\prime}, X \backslash S\right)$ is $\delta$-extendable for $J$. That is $C^{\prime}$ is a set of cluster centers. Moreover $C^{\prime}$ can be extended to a $(1+\delta)$-approximate solution with $X \backslash S$ being assigned to clusters corresponding to $C^{\prime}$. Initially we set our set of partial solution to be $\mathcal{P}=\{(\emptyset, X, 0)\}$. Clearly $(\emptyset, X, 0)$ is a good partial solution. At each iteration we maintain an invariant that $\mathcal{P}$ contains a good partial solution with high probability. At any step if there is a partial solution $\left(C_{1}, S, \delta\right) \in \mathcal{P}$ such that $\left|C_{1}\right|<k$ and $S \neq \emptyset$ we do the following. First we delete $\left(C_{1}, S, \delta\right)$ from $\mathcal{P}$ and then we add following partial solutions to $\mathcal{P}$. Let $\mathbf{v}_{1}, \ldots, \mathbf{v}_{|S|}$ be the vectors in $S$ ordered according to the non-decreasing order of $d_{H}\left(\mathbf{v}_{i}, C_{1}\right)$.

(i) We add $\left(C_{1}, S^{\prime}, \delta\right)$, where $S^{\prime}$ is the last $\left\lceil\frac{|S|}{2}\right\rceil$ vectors in the order $\mathbf{v}_{1}, \ldots, \mathbf{v}_{|S|}$.

(ii) For any choice of $k^{\prime} \in\left\{1, \ldots, k-\left|C_{1}\right|\right\}$ we extend the set $C_{1}$ assuming $\left(C_{1}, X \backslash S\right)$ is $\delta$-extendable (see the below paragraph for a brief explanation).

Assume that $C_{2}$ is a good $\delta$-extension of $\left(C_{1}, X \backslash S\right)$ (see Definition 11). Since $C_{2}$ is a good $\delta$-extension of $\left(C_{1}, X \backslash S\right)$, there exist $I \in\left(\begin{array}{c}{[k]} \\ \left|C_{1}\right|\end{array}\right)$ and a partition $Z_{1} \uplus Z_{2}$ of $S$ such that $C_{2}$ is an optimum solution to $J^{\prime}=\left(Z_{2}, \ell=k-\left|C_{1}\right|, \mathcal{R}\left(I, C_{1}\right)\right)$ and $\operatorname{cost}\left(S, C_{1} \cup C_{2}\right)=$ $\operatorname{cost}\left(Z_{1}, C_{1}\right)+\operatorname{cost}\left(Z_{2}, C_{2}\right)$ (see Lemma 8). Let $B^{\prime}$ be the set defined in Lemma 7. If $\left|B^{\prime}\right| \geq\left\lfloor\frac{|S|}{2}\right\rfloor$, then by Lemma $7(i i)$ and Observation $6,\left(C_{1}, S^{\prime}, \delta\right)$ is $\delta$-extendable for $J$ (this is covered in item $(i)$ above). Suppose the size of $B^{\prime}$ is at most $\left\lfloor\frac{|S|}{2}\right\rfloor$. Then, by Lemma $7($ iii), we know that $\left|Z_{2}\right|$ is a constant fraction of $|S|$. Therefore the size of the largest cluster among the clusters correspond to $C_{2}$ in the instance $J^{\prime}$ is also a constant fraction of $|S|$. Let $X_{1}, \ldots, X_{\ell}$ be the clusters corresponding to the solution $C_{2}$, where $\ell=\left|C_{2}\right|$. For the ease of presentation assume that $\left|X_{1}\right| \geq\left|X_{2}\right| \ldots \geq\left|X_{\ell}\right|$. Let $k^{\prime}$ be the smallest integer such that $\left|X_{k^{\prime}}\right|$ is "much larger than" $\left|X_{k^{\prime}+1}\right|$. In other words, for any $i \in\left\{1, \ldots, k^{\prime}-1\right\}$, the size of $X_{i}$ is at most a constant times $\left|X_{i+1}\right|$. This implies that the size of $X_{j}$ is a constant fraction of $|S|$ for any $j \in\left\{1, \ldots, k^{\prime}\right\}$. Therefore we use Lemma 5 to compute an approximate set of centers $C^{\prime}$ for the clusters $X_{1}, \ldots, X_{j}$. Then we add $\left(C_{1} \cup C^{\prime}, S, 5 \delta+4 \alpha\right)$ to $\mathcal{P}$ (this case is covered in item (ii) above). The explanation for setting the parameter $\alpha$ and how to estimate weights $w_{i}$ used in Lemma 5 is given in the next paragraph. At the end, for any tuple $(C, S, \delta) \in \mathcal{P}$, either $|C|=k$ or $S=\emptyset$. Then we output the best solution among all the tuples in $\mathcal{P}$. The correctness of the algorithm will follow from the invariant that $\mathcal{P}$ contains a good partial solution with high probability at each iteration.

Now we explain how to set different parameters for the algorithm, which are used in Lemmas 5,7 , and 9. We have an assumption that $J$ is $\left(k, \frac{\varepsilon}{8 k^{*}}\right)$-irreducible. In Lemmas 7 and 9, we want $J$ to be $\left(k, 5 \delta^{\prime}\right)$-irreducible. So we set $\delta^{\prime}=\frac{\varepsilon}{40 k^{*}}$. Initially we set $\mathcal{P}=\{(\emptyset, X, 0)\}$. At each iteration we extend a partial solution $\left(C_{1}, S, \delta_{1}\right)$ either by deleting half of vectors from $S$ or by computing a set of center vectors using Lemma 5 and the correctness of the step (assuming $\left(C_{1}, X \backslash S\right)$ is $\delta_{1}$-extendable) follows from Lemma 9 . For the initial application of Lemma 9 (i.e., where $\left.\left(C_{1}, S, \delta_{1}\right)=(\emptyset, X, 0)\right)$, we have that $\delta_{1}=0$. Then after each application of Lemma 9 we get a partial solution which we expect to be $\left(5 \delta_{1}+4 \alpha\right)$-extendable (we fix $\alpha$ later). The number of times Lemma 9 is applied to get a particular solution in $\mathcal{P}$ (at the end of the algorithm) is at 
most $k$. This implies that at the end some solution in $\mathcal{P}$ is $\gamma(k)$-extendable (by Observation 6 ), where $\gamma(k)$ can be obtained from the following recurrence relation.

$$
\begin{aligned}
& \gamma(0)=0, \text { and } \\
& \gamma\left(k^{\prime}\right)=5 \gamma\left(k^{\prime}-1\right)+4 \alpha, \text { for } k^{\prime} \in\{1,2, \ldots, k\} .
\end{aligned}
$$

The above recurrence relation solves to $\gamma\left(k^{\prime}\right)=\left(5^{k^{\prime}}-1\right) \alpha$ for any $k^{\prime} \in\{0,1, \ldots, k\}$. Moreover, by Lemma 9 , we need $\gamma\left(k^{\prime}\right)$ to be at most $\delta^{\prime}$ for all $k^{\prime} \in\{0,1, \ldots, k\}$. Thus we set $\alpha=\frac{\delta^{\prime}}{\left(5^{k}-1\right)}$. From Lemma 5 , we expect a $(1+\alpha)$-approximate solution for a restricted instance derived from $J$. So we use $\varepsilon=\frac{\alpha}{7}$ and $\delta=\frac{\alpha}{7}$ in the application of Lemma 5. To apply Lemma 5, we also need the value for $\beta$ and promised bounds on the sizes of the clusters for which we are seeking center vectors. Towards that we give a detailed explanation of how to use Lemma 5. Notice that $\left(C_{1}, S, \delta_{1}\right)$ is a partial solution already computed and we assumed that $\left(C_{1}, X \backslash S\right)$ is $\delta_{1}$ extendable for $J$. Let $C_{2}$ is a good $\delta$-extension of $\left(C_{1}, X \backslash S\right)$. Since $C_{2}$ is a good $\delta$-extension of $\left(C_{1}, X \backslash S\right)$, there exist $I \in\left(\begin{array}{c}{[k]} \\ \left|C_{1}\right|\end{array}\right)$ and a partition $Z_{1} \uplus Z_{2}$ of $S$ such that $C_{2}$ is an optimum solution to $J^{\prime}=\left(Z_{2}, \ell=k-\left|C_{1}\right|, \mathcal{R}\left(I, C_{1}\right)\right)$ and $\operatorname{cost}\left(S, C_{1} \cup C_{2}\right)=\operatorname{cost}\left(Z_{1}, C_{1}\right)+\operatorname{cost}\left(Z_{2}, C_{2}\right)$ (see Lemma 8). Let $B^{\prime}$ be the set defined in Lemma 7 . The application of Lemma 5 is important for the partial solution $\left(C_{1}, S, \delta_{1}\right)$ only when the size of $B^{\prime}$ is at most $\frac{|S|}{2}$. Then, by Lemma $7(i i i)$, we know that $\left|Z_{2}\right| \geq\left(\frac{\delta^{\prime}}{2\left(1+\delta^{\prime}\right)}\right)|S|$. Therefore, the largest cluster corresponding to the solution $C_{2}$ of $J^{\prime}$ is at least $\left(\frac{\delta^{\prime}}{2 k\left(1+\delta^{\prime}\right)}\right)|S|$. Let $X_{1}, \ldots, X_{\ell}$ be the clusters corresponding $C_{2}$, where $\ell=\left|C_{2}\right|$. For the ease of presentation assume that $\left|X_{1}\right| \geq\left|X_{2}\right| \ldots \geq\left|X_{\ell}\right|$. Let $k^{\prime}$ be the smallest integer such that $\left|X_{k^{\prime}}\right|>\frac{k}{\alpha}\left|X_{k^{\prime}+1}\right|$. Therefore we have that $\left|X_{1}\right| \leq \frac{k}{\alpha}\left|X_{2}\right| \leq \ldots \leq$ $\left(\frac{k}{\alpha}\right)^{k^{\prime}-1}\left|X_{k^{\prime}}\right|$ (Here the set $\left[k^{\prime}\right]$ plays the role of $I^{\prime}$ in Lemma 9, i.e., $\left[k^{\prime}\right]$ is a set of indices of $\frac{k}{\alpha}$-heavy clusters). This implies that the size of $X_{j}$ is at least $\left(\frac{\alpha}{k}\right)^{k^{\prime}-1}\left|X_{1}\right| \geq\left(\frac{\alpha}{k}\right)^{k-1} \frac{\delta^{\prime}}{2 k\left(1+\delta^{\prime}\right)}|S|$ (because $\frac{\alpha}{k} \leq 1$ ) for any $j \in\left[k^{\prime}\right]$. So we set $\beta=\left(\frac{\alpha}{k}\right)^{k-1} \frac{\delta^{\prime}}{k\left(2+\delta^{\prime}\right)}$. We set the value $c$ in Lemma 5 to be $\left|X_{k^{\prime}}\right|$. Notice that we do not have to know the value of $c$ explicitly, but instead we need to know the weights $w_{i}$ s within a promised bound. From the value of $c$ it is clear that for any $j \in\left[k^{\prime}\right], \frac{\left|X_{j}\right|}{c} \leq\left(\frac{k}{\alpha}\right)^{k^{\prime}-j} \leq\left(\frac{k}{\alpha}\right)^{k^{\prime}-1}$. Let $h=\left(\frac{k}{\alpha}\right)^{k^{\prime}-1}$. Then there exists $w_{j} \in\left\{(1+\delta)^{0},(1+\delta)^{1} \ldots,(1+\delta)^{\log _{1+\delta} h}\right\}$ such that $\frac{\left|X_{j}\right|}{c} \leq w_{j} \leq \frac{(1+\delta)\left|X_{j}\right|}{c}$. Thus we apply Lemma 5 for all possible values $w_{1}, \ldots, w_{k^{\prime}} \in\left\{(1+\delta)^{0},(1+\delta)^{1} \ldots,(1+\delta)^{\log _{1+\delta} h}\right\}$ and extend the set $C_{1}$ in each case.

Now we are ready to give the formal proof of the theorem.

Proof of Theorem 9. The pseudocode of our algorithm is given in Algorithm 1. First, we define an iteration of the algorithm to be the execution of one step of the for loop at Line 3 . That is at the beginning of an iteration one partial solution will be deleted from $\mathcal{P}$ and later during the execution of the iteration many partial solutions will be added to $\mathcal{P}$ (see Lines 4 and 15). Next, we prove that the algorithm terminates. Notice that when there is no partial solution $\left(C_{1}, S, \delta_{1}\right) \in \mathcal{P}$ with $\left|C_{1}\right|<k$ and $S \neq \emptyset$, then the algorithm terminates. When there is a partial solution $\left(C_{1}, S, \delta_{1}\right) \in \mathcal{P}$ with $\left|C_{1}\right|<k$ and $S \neq \emptyset$, then there is an iteration of the algorithm (Line 3) where in the for loop we consider $\left(C_{1}, S, \delta_{1}\right)$, delete $\left(C_{1}, S, \delta_{1}\right)$ from $\mathcal{P}$ and add many partial solutions to $\mathcal{P}$. For each such partial solution $\left(C_{1}^{\prime}, S^{\prime}, \delta_{2}\right)$, either $\left|C_{1}^{\prime}\right|>\left|C_{1}\right|$ or $\left|S^{\prime}\right|<|S|$. This implies that Algorithm 1 will terminate.

Now we prove the correctness of the algorithm. That is we prove that Algorithm 1 will return a $\left(1+\frac{\varepsilon}{40 k^{*}}\right)$-approximate solution with probability at least $p\left(k^{*}, k, \varepsilon\right)$.

For convenience we let the 0th iteration to be the initial assignments before any of the execution of Line 3. That is at the end of the 0 th iteration we have that $\mathcal{P}=\{(\emptyset, X, 0)\}$. When we apply in line 14 Algorithm $\mathcal{A}$ from Lemma 5 on input $S, k^{\prime}, \operatorname{proj}_{I^{\prime}}\left(\mathcal{R}^{\prime}\right), \beta, \delta, \varepsilon$, and 




$w_{1}, \ldots, w_{k^{\prime}}$, we know that by Lemma 5 , it outputs a solution with probability at least $\frac{\varepsilon \cdot \beta^{r \cdot k}}{1+\varepsilon}$, where $r=\Theta\left(\frac{k}{\varepsilon^{2}} \log \frac{1}{\varepsilon}\right)$. Let $c^{\prime}$ be a constant such that $r=c^{\prime} \cdot \frac{k}{\varepsilon^{2}} \log \frac{1}{\varepsilon}$.

Correctness Invariant: At the end of every iteration of the algorithm, there is a partial solution $\left(C, S, \delta_{1}\right) \in \mathcal{P}$ such that $(C, X \backslash S)$ is $\delta_{1}$-extendable for $J$ with probability at least $\left(\frac{\varepsilon \beta^{r}}{1+\varepsilon}\right)^{q}$ where $q=|C|$.

We need the following observation to prove the correctness invariant.

Observation 10. At any step of the algorithm, for any partial solution $\left(C, S, \delta_{1}\right) \in \mathcal{P}, \delta_{1} \leq \delta^{\prime}$.

Proof Sketch. By induction on the number of iteration of the algorithm one can prove that at the end of each iteration, for any partial solution $\left(C, S, \delta_{1}\right) \in \mathcal{P}, \delta_{1} \leq \gamma(|C|)$. Recall that $\gamma\left(k^{\prime}\right)=\left(5^{k^{\prime}}-1\right) \alpha$ and $\gamma\left(k^{\prime}\right) \leq \delta^{\prime}$ for all $k^{\prime} \in\{0,1, \ldots, k\}$. Thus the observation follows.

Claim 7. Correctness invariant is maintained at the end of every iteration.

Proof. We prove the claim using induction on the number of iterations. The base case is for the 0 th iteration. Since at the end of 0 th iteration $(\emptyset, X, 0) \in \mathcal{P}$ and the fact that $(\emptyset, \emptyset)$ is 0 -extendable for $J$ with probability 1 , the base case follows. Now we consider the induction 
step. That is consider the iteration $i$ of the algorithm where $i>0$. Let $\left(C_{1}, S, \delta^{*}\right)$ be the partial solution for which the iteration $i$ corresponds to. At the beginning of iteration $i,\left(C_{1}, S, \delta^{*}\right) \in \mathcal{P}$ and by induction hypothesis there is a partial solution $\left(C^{\prime}, S^{\prime}, \delta_{1}\right) \in \mathcal{P}$ satisfying the properties mentioned in the correctness invariant. Notice that during the iteration $i$, we will delete $\left(C_{1}, S, \delta^{*}\right)$ from $\mathcal{P}$ and add many partial solutions to $\mathcal{P}$. If $\left(C^{\prime}, S^{\prime}, \delta_{1}\right) \neq\left(C_{1}, S, \delta^{*}\right)$ then at the end of iteration $i,\left(C^{\prime}, S^{\prime}, \delta_{1}\right) \in \mathcal{P}$ and the invariant follows.

So now we assume that $\left(C_{1}, S, \delta^{*}\right)=\left(C^{\prime}, S^{\prime}, \delta_{1}\right)$. This implies that $\left(C_{1}, X \backslash S\right)$ is $\delta^{*}$ extendable for $J$ with probability at least $\left(\frac{\varepsilon \beta^{r}}{1+\varepsilon}\right)^{q}$, where $q=\left|C_{1}\right|$. By Lemma 8 , there is a good $\delta^{*}$-extension $C_{2}$ of $\left(C_{1}, X \backslash S\right)$, a partition $Z_{1} \uplus Z_{2}$ of $X \backslash S$ and $I \in\left(\begin{array}{c}k \\ \left|C_{1}\right|\end{array}\right)$ such that $C_{1} \in \operatorname{proj}_{I}(\mathcal{R})$ and $C_{2}$ is an optimum solution to $\left(Z_{2}, k-\left|C_{1}\right|, \mathcal{R}\left(I, C_{1}\right)\right)$ and $\operatorname{cost}\left(S, C_{1} \cup C_{2}\right)=$ $\operatorname{cost}\left(Z_{1}, C_{1}\right)+\operatorname{cost}\left(Z_{2}, C_{2}\right)$. Let $t=\min _{\mathbf{c} \in C_{1}, \mathbf{c}^{\prime} \in C_{2}} d_{H}\left(\mathbf{c}, \mathbf{c}^{\prime}\right)$. Let $B^{\prime}=\bigcup_{\mathbf{c} \in C_{1}} \mathcal{B}\left(\mathbf{c}, t^{\prime} / 2\right) \cap S$ and $S^{*}=S \backslash B^{\prime}$. Then by Lemma 7 , we get the following.

(i) $B^{\prime} \subseteq Z_{1}$ and $B^{\prime}$ consists of the first $\left|B^{\prime}\right|$ vectors of $S$ in the ordering according to the non-decreasing distance $d_{H}\left(\mathbf{x}, C_{1}\right)$ where $\mathbf{x} \in S$.

(ii) $\operatorname{cost}\left(S^{*}, C_{1} \cup C_{2}\right)=\operatorname{cost}\left(Z_{1} \backslash B^{\prime}, C_{1}\right)+\operatorname{cost}\left(Z_{2}, C_{2}\right)$. Moreover, $\left(C_{1}, X \backslash S^{*}\right)$ is $\delta^{*}$-extendable for $J$ and $C_{2}$ is a $\delta^{*}$-extension of $\left(C_{1}, X \backslash S^{*}\right)$.

(iii) Since $\delta^{*} \leq \delta^{\prime}$ (by Observation 10) and the fact that $J$ is $\left(k, 5 \delta^{\prime}\right)$-irreducible (because of our assumption), we have that $\left|Z_{2}\right| \geq\left(\frac{\delta^{\prime}}{1+\delta^{\prime}}\right)\left|S^{*}\right|$. If $\left|B^{\prime}\right| \leq \frac{|S|}{2}$, then $\left|Z_{2}\right| \geq\left(\frac{\delta^{\prime}}{2\left(1+\delta^{\prime}\right)}\right)|S|$.

Suppose $\left|B^{\prime}\right|>\frac{|S|}{2}$. Recall the definition of $S^{\prime}$ from Line 6. Notice that $S \backslash S^{\prime} \subseteq B^{\prime}$. Therefore, by the assumption that $\left(C_{1}, X \backslash S\right)$ is $\delta^{*}$-extendable for $J$ and by Lemma $7(i i)$ and Observation $6,\left(C_{1}, X \backslash S^{\prime}\right)$ is $\delta^{*}$-extendable for $J$. Since $\left(C_{1}, X \backslash S\right)$ is $\delta^{*}$-extendable with probability at least $\left(\frac{\varepsilon \beta^{r}}{1+\varepsilon}\right)^{q},\left(C_{1}, X \backslash S^{\prime}\right)$ is $\delta^{*}$-extendable with probability at least $\left(\frac{\varepsilon \beta^{r}}{1+\varepsilon}\right)^{q}$.

Now consider the case $\left|B^{\prime}\right| \leq \frac{|S|}{2}$. We know that $C_{2}$ is an optimum solution to $\left(Z_{2}, \ell=k-\right.$ $\left.\left|C_{1}\right|, \mathcal{R}\left(I, C_{1}\right)\right)$. Let $C_{2}=\left\{\mathbf{c}_{1}, \ldots, \mathbf{c}_{\ell}\right\}$ and $Y_{1}, \ldots Y_{\ell}$ be the clusters corresponding to the solution $C_{2}$ of $\left(Z_{2}, \ell=k-\left|C_{1}\right|, \mathcal{R}\left(I, C_{1}\right)\right)$. Let $\pi$ be a permutation of $[\ell]$ such that $\left|Y_{\pi(1)}\right| \geq \ldots \geq\left|Y_{\pi(\ell)}\right|$. Let $X_{j}=Y_{\pi(j)}$ and $\mathbf{c}_{j}^{*}=\mathbf{c}_{\pi(j)}$ for all $j \in[\ell]$. Let $k^{\prime}$ be the smallest integer in $[\ell]$ such that $\left|X_{k^{\prime}}\right|>\left(\frac{k}{\alpha}\right)\left|X_{k^{\prime}+1}\right|$. This implies that $\left|X_{1}\right| \leq\left(\frac{k}{\alpha}\right)\left|X_{2}\right| \leq \ldots \leq\left(\frac{k}{\alpha}\right)^{k^{\prime}-1}\left|X_{k^{\prime}}\right|$. Thus, by the fact that $\left|X_{1}\right| \geq \frac{\left|Z_{2}\right|}{\ell} \geq \frac{\left|Z_{2}\right|}{k}$, we have that for any $j \in\left[k^{\prime}\right]$,

$$
\left|X_{j}\right| \geq\left(\frac{\alpha}{k}\right)^{k^{\prime}-1} \frac{\left|Z_{2}\right|}{k} .
$$

Let $I^{\prime}=\left\{\pi(j): j \in\left[k^{\prime}\right]\right\}$. By statement (iii) above and (34), for all $j \in I^{\prime}$,

$$
\left.\left|Y_{j}\right| \geq\left(\frac{\alpha}{k}\right)^{k^{\prime}-1} \cdot \frac{\delta^{\prime}}{2 k\left(1+\delta^{\prime}\right)}|S| \geq\left(\frac{\alpha}{k}\right)^{k-1} \cdot \frac{\delta^{\prime} \cdot|S|}{2 k\left(1+\delta^{\prime}\right)} \quad \text { (Since } \frac{\alpha}{k} \leq 1\right)
$$

This implies that for all $j \in I^{\prime},\left|Y_{j}\right| \geq \beta|S|$. Let $C^{*}=\left\{\mathbf{c}_{j}: j \in I^{\prime}\right\}$. Let $V=\sum_{j \in I^{\prime}} \operatorname{cost}\left(Y_{j}, \mathbf{c}_{j}\right)$. Moreover, (a) $\left\{Y_{j}: j \in I^{\prime}\right\}$ is a set of $\frac{k}{\alpha}$-heavy clusters (see Definition 12). Let $c=\left|Y_{k^{\prime}}\right|$. Notice that $(b)$ for any $j \in I^{\prime}, \frac{Y_{j}}{c} \leq\left(\frac{k}{\alpha}\right)^{k^{\prime}-1}$. Let $\mathcal{R}^{\prime}=\mathcal{R}\left(I, C_{1}\right)$ and $J^{\prime}$ be the instance $(Z=$ $\left.\bigcup_{j \in I^{\prime}} Y_{j}, k^{\prime}, \operatorname{proj}_{I^{\prime}}\left(\mathcal{R}^{\prime}\right)\right)$. Now in the iteration $i$, consider the execution of for loop at Line 11 for $k^{\prime}$ and $I^{\prime}$. Notice that for any $j \in I^{\prime}$, there exists $w \in\left\{(1+\delta)^{0},(1+\delta)^{1} \ldots,(1+\delta)^{\log _{1+\delta} h}\right\}$, where $h=\left(\frac{k}{\alpha}\right)^{k^{\prime}-1}$, such that $\frac{\left|Y_{j}\right|}{c} \leq w \leq \frac{(1+\delta)\left|Y_{j}\right|}{c}$. Now consider the for loop at Line 13 for values $w_{1}, \ldots, w_{k^{\prime}}$ such that $\frac{\left|Y_{\pi(j)}\right|}{c} \leq w_{j} \leq \frac{(1+\delta)\left|Y_{\pi(j)}\right|}{c}$ for all $j \in\left[k^{\prime}\right]$. Then, by Lemma 5 , in 
Line 14 we get a set $C^{\prime}=\left\{\mathbf{c}_{i}^{\prime}: i \in I^{\prime}\right\}$ of $k^{\prime}$ cluster centers such that with probability at least $\frac{\varepsilon \cdot \beta^{r \cdot k^{\prime}}}{1+\varepsilon}$

$$
\sum_{i \in I^{\prime}} \operatorname{cost}\left(Y_{i},\left\{\mathbf{c}_{i}^{\prime}\right\}\right) \leq(1+\varepsilon)^{2}(1+\delta) V \leq(1+\alpha) \cdot \sum_{j \in I^{\prime}} \operatorname{cost}\left(Y_{j}, \mathbf{c}_{j}\right) .
$$

Therefore, $\left(C_{1} \cup C^{\prime}, S, 5 \delta^{*}+4 \alpha\right)$ belongs to $\mathcal{P}$ at the end of iteration $i$ with probability at least $\left(\frac{\varepsilon \cdot \beta^{r}}{1+\varepsilon}\right)^{\left(q+k^{\prime}\right)}=\left(\frac{\varepsilon \cdot \beta^{r}}{1+\varepsilon}\right)^{\left|C_{1} \cup C^{\prime}\right|}$. Then by Lemma $9,\left(C_{1} \cup C^{\prime}, X \backslash S\right)$ is $\left(5 \delta^{*}+4 \alpha\right)$-exdendable for $J$ and this completes the proof of the claim.

Now for the proof of the correctness of the algorithm consider the invariant at the end of the last iteration. At the end of last iteration for all $\left(C, S, \delta_{1}\right) \in \mathcal{P}$, we have that either $|C|=k$ or $S=\emptyset$. Then by Claim 7 , at the end of the last iteration, $\mathcal{P}$ contains a partial solution $\left(C, S, \delta_{1}\right)$ which is $\delta_{1}$-extendable with probability at least

$$
p=\left(\frac{\varepsilon \beta^{r}}{1+\varepsilon}\right)^{k} .
$$

Then the output set $D \supseteq C$ is a $\left(1+\delta_{1}\right)$-approximate solution of $J$ with probability at least $p$, by Proposition 5. By Observation $10, D$ is a $\left(1+\frac{\varepsilon}{40 k^{*}}\right)$-approximate solution of $J$. By substituting the value of $\beta$ and $r$ into (37), we bound the value of $p$ as follows.

$$
\begin{aligned}
p & =\left(\frac{\varepsilon}{1+\varepsilon}\right)^{k} \cdot\left(\left(\frac{\delta^{\prime}}{k\left(5^{k}-1\right)}\right)^{k-1} \frac{\delta^{\prime}}{2 k\left(1+\delta^{\prime}\right)}\right)^{r \cdot k} \\
& =\left(\frac{\varepsilon}{1+\varepsilon}\right)^{k} \cdot\left(\frac{\left(\frac{\varepsilon}{40 k^{*}}\right)^{k}}{\left(k\left(5^{k}-1\right)\right)^{k-1} \cdot 2 k \cdot\left(1+\frac{\varepsilon}{40 k^{*}}\right)}\right)^{r \cdot k} \\
& =\left(\frac{\varepsilon}{1+\varepsilon}\right)^{k} \cdot\left(\frac{\varepsilon^{k}}{\left(40 k \cdot k^{*} \cdot\left(5^{k}-1\right)\right)^{k-1} \cdot 2 k \cdot\left(40 k^{*}+\varepsilon\right)}\right)^{r \cdot k} \\
& =\left(\frac{\varepsilon}{1+\varepsilon}\right)^{k} \cdot\left(\frac{\varepsilon^{k}}{\left(40 k \cdot k^{*} \cdot\left(5^{k}-1\right)\right)^{k-1} \cdot 2 k \cdot\left(40 k^{*}+\varepsilon\right)}\right)^{\frac{c^{\prime} \cdot k^{2}}{\varepsilon^{2}} \log \frac{1}{\varepsilon}}
\end{aligned}
$$

Running time. Now we analyze the running time of the algorithm. Notice that in the algorithm initially we have $\mathcal{P}=\{(\emptyset, X, 0)\}$ and in each step we delete a partial solution from $\mathcal{P}$ and add many partial solutions. Towards analysing the running time we define a node-labelled rooted tree $T$ as follows. The root is labelled with $(\emptyset, X, 0)$. Each node of the tree is labelled with a partial solution $\left(C, S, \delta_{1}\right)$, which corresponds an execution of for loop at Line 3 . For a node labelled with $\left(C, S, \delta_{1}\right)$, let $S_{1}, \ldots, S_{\ell}$ be the tuples added to $\mathcal{P}$ during the iteration corresponding to $\left(C, S, \delta_{1}\right)$. Then the node labelled $\left(C, S, \delta_{1}\right)$ will have $\ell$ children and they are labelled with $S_{1}, \ldots, S_{\ell}$, respectively. Therefore, the number of iterations in the algorithm is equal to the number of nodes in the tree $T$. In each iteration (corresponding to a partial solution $\left.\left(C, S, \delta_{1}\right)\right)$ we sort the set $S$ of vectors according to the Hamming distance to $C$ (see Line 5). This can be done in time $\mathcal{O}\left(k n^{\prime} m\right)$, where $n^{\prime}=|S|$. Then we add $\left(C, S^{\prime}, \delta_{1}\right)$ to $\mathcal{P}$, where $\left|S^{\prime}\right| \leq \frac{|S|}{2}$. Then because of Lines 8,11 and 13 , we add at most $L=2^{2 k} \cdot\left(\log _{1+\delta} \frac{k}{\alpha}\right)^{k}$ tuples to $\mathcal{P}$ (in Line 14) where the cardinality of the first entry of the each tuple is strictly more that $|C|$. The time required to execute the Line 14 is at most $\mathcal{O}\left(m 2^{k}\left(\frac{k}{\varepsilon}\right)^{2} \log \frac{1}{\varepsilon}\right)$ (by Lemma 5). Therefore the time spend in one iteration of the algorithm is at most

$$
\mathcal{O}\left(L \cdot 2^{k} n^{\prime} \cdot m \cdot\left(\frac{k}{\varepsilon}\right)^{2} \log \frac{1}{\varepsilon}\right) .
$$


For any node $v$ labelled with $\left(C, S, \delta_{1}\right)$, let $N(k-|C|,|S|)$ be the time taken by the iterations which are labelled by the nodes of the subtree of $T$, rooted at the node $v$. The value of $N(k-|C|,|S|)$ can be upper bounded using the following recurrence formula. There is a constant $c$ such that for any $0 \leq k^{\prime} \leq k$ and $0 \leq n^{\prime} \leq n$,

$N\left(k^{\prime}, n^{\prime}\right) \leq\left\{\begin{array}{cc}c & \text { if } \\ N\left(k^{\prime}+\frac{n^{\prime}}{2}\right)+L \cdot N\left(k^{\prime}-1, n^{\prime}\right)+\left(c L \cdot 2^{k} n^{\prime} m\left(\frac{k}{\varepsilon}\right)^{2} \log \frac{1}{\varepsilon}\right) & \text { if } n^{\prime}=0 \\ k^{\prime}, n^{\prime}>0\end{array}\right.$

Clearly, the running time of the algorithm will be upper bounded by $N(k, n)$. We claim that

$$
N(k, n) \leq c \cdot(2 L)^{k} 2^{3 k^{2}} \cdot n \cdot m \cdot\left(\frac{k}{\varepsilon}\right)^{2} \log \frac{1}{\varepsilon} .
$$

Towards that we prove that for any $0 \leq k^{\prime} \leq k$ and $0 \leq n^{\prime} \leq n, N\left(k^{\prime}, n^{\prime}\right) \leq c \cdot 2^{k} \cdot L^{k^{\prime}} 2^{3 k^{\prime 2}} \cdot n^{\prime}$. $m\left(\frac{k}{\varepsilon}\right)^{2} \log \frac{1}{\varepsilon}$ using induction. The base case is when $k^{\prime}=0$ or $n^{\prime}=0$ and it holds by (38). By induction hypothesis we have that

$$
\begin{aligned}
N\left(k^{\prime}, n^{\prime}\right) \leq\left(c \cdot 2^{k} L^{k^{\prime}} 2^{3 k^{\prime 2}} \frac{n^{\prime} m}{2}\left(\frac{k}{\varepsilon}\right)^{2} \log \frac{1}{\varepsilon}\right) & +\left(L \cdot c 2^{k} L^{k^{\prime}-1} 2^{3\left(k^{\prime}-1\right)^{2}} n^{\prime} m\left(\frac{k}{\varepsilon}\right)^{2} \log \frac{1}{\varepsilon}\right) \\
& +\left(c L \cdot 2^{k} n^{\prime} \cdot m\left(\frac{k}{\varepsilon}\right)^{2} \log \frac{1}{\varepsilon}\right) .
\end{aligned}
$$

To prove that $N\left(k^{\prime}, n^{\prime}\right) \leq c 2^{k} \cdot L^{k^{\prime}} 2^{3 k^{\prime 2}} \cdot n^{\prime} \cdot m \cdot\left(\frac{k}{\varepsilon}\right)^{2} \log \frac{1}{\varepsilon}$, it is enough to prove that $2^{3 k^{\prime 2}-1}+$ $2^{3\left(k^{\prime}-1\right)^{2}}+1 \leq 2^{3 k^{\prime 2}}$, which is true for any $k^{\prime} \geq 1$.

Therefore we upper bound the running time of the algorithm as follow.

$$
\begin{aligned}
N(k, n) & \leq\left(c 2^{k} 2^{3 k^{2}} \cdot n \cdot m\left(\frac{k}{\varepsilon}\right)^{2} \log \frac{1}{\varepsilon}\right) \cdot L^{k} \\
& \leq\left(2^{\mathcal{O}\left(k^{2}\right)} \cdot n \cdot m\left(\frac{1}{\varepsilon}\right)^{2} \log \frac{1}{\varepsilon}\right) \cdot 2^{2 k} \cdot\left(\log _{1+\delta} \frac{k}{\alpha}\right)^{k} \\
& \leq\left(2^{\mathcal{O}\left(k^{2}\right)} \cdot n \cdot m\left(\frac{1}{\varepsilon}\right)^{2} \log \frac{1}{\varepsilon}\right) \cdot\left(\frac{\ln \frac{k}{\alpha}}{\ln (1+\delta)}\right)^{k} \\
& \leq\left(2^{\mathcal{O}\left(k^{2}+k \log \left(k^{*}\right)\right)} \cdot n \cdot m \cdot \frac{1}{\varepsilon^{2}} \log \frac{1}{\varepsilon}\right) \cdot\left(\frac{\ln \frac{1}{\varepsilon}}{\ln (1+\delta)}\right)^{k} \\
& \leq\left(2^{\mathcal{O}\left(k^{2}+k \log \left(k^{*}\right)\right)} \cdot n \cdot m \cdot \frac{1}{\varepsilon^{2}} \log \frac{1}{\varepsilon}\right) \cdot\left(\frac{(1+\delta) \ln \frac{1}{\varepsilon}}{\delta}\right)^{k} \\
& \leq\left(2^{\mathcal{O}\left(k^{2}+k \log \left(k^{*}\right)\right)} \cdot n \cdot m\right) \cdot\left(\frac{1}{\varepsilon}\right)^{\mathcal{O}(k)} \cdot
\end{aligned}
$$

This completes the proof of the theorem.

\subsection{The final step}

Now we are ready to prove Theorem 2 . 
Let $J=(X, k, \mathcal{R})$ be the input instance. Let

$$
p\left(k^{*}, k, \varepsilon\right)=\left(\frac{\varepsilon}{1+\varepsilon}\right)^{k} \cdot\left(\frac{\varepsilon^{k}}{\left(40 k \cdot k^{*} \cdot\left(5^{k}-1\right)\right)^{k-1} \cdot 2 k \cdot\left(40 k^{*}+\varepsilon\right)}\right)^{\frac{c^{\prime} \cdot k^{2}}{\varepsilon^{2}} \log \frac{1}{\varepsilon}}
$$

be the success probability from Theorem 9 , where $c^{\prime}$ is a constant. For each $I \subseteq[k]$, we apply Theorem 9 on $\left(X,|I|, \operatorname{proj}_{I}(\mathcal{R})\right)$ and $\frac{\varepsilon}{4}$ (where we substitute $k=|I|$ and $\left.k^{*}=k\right) \frac{1}{p\left(k,|I|, \frac{\varepsilon}{4}\right)}$ times. Let $C_{I}^{\prime}$ be the best solution obtained by the above process. By using Proposition 5 , let $C_{I}$ be the solution for $J$ obtained from $C_{I}^{\prime}$. Then we output the best solution among $\left\{C_{I}: I \subseteq[k]\right\}$. The running time of the algorithm mentioned in Theorem 9 is $2^{\mathcal{O}\left(k^{2}+k \log \left(k^{*}\right)\right)} \cdot n \cdot m \cdot\left(\frac{1}{\varepsilon}\right)^{\mathcal{O}(k)}$ and the running time of the algorithm mentioned in Proposition 5 is linear in the input size. Thus the running time of our algorithm is at most $\frac{1}{p\left(k, k, \frac{\varepsilon}{4}\right)} \cdot 2^{\mathcal{O}\left(k^{2}\right)} \cdot\left(\frac{1}{\varepsilon}\right)^{\mathcal{O}(k)} \cdot n \cdot m$. The value of $\frac{1}{p\left(k, k, \frac{\varepsilon}{4}\right)}$ is upper bounded as follows.

$$
\begin{aligned}
\frac{1}{p\left(k, k, \frac{\varepsilon}{4}\right)} & =\left(\frac{4+\varepsilon}{\varepsilon}\right)^{k} \cdot\left(\frac{\left(40 k^{2} \cdot\left(5^{k}-1\right)\right)^{k-1} \cdot 2 k \cdot\left(40 k+\frac{\varepsilon}{4}\right)}{\left(\frac{\varepsilon}{4}\right)^{k}}\right)^{\frac{\frac{c}{}^{\prime} \cdot k^{2}}{\varepsilon^{2}} \log \frac{1}{\varepsilon}} \\
& =\left(\frac{1}{\varepsilon}\right)^{\mathcal{O}(k)} \cdot 2^{\mathcal{O}\left(\frac{k^{4}}{\varepsilon^{2}} \log \frac{1}{\varepsilon}\right)} \cdot\left(\frac{1}{\varepsilon}\right)^{\mathcal{O}\left(\frac{k}{\varepsilon^{2}} \log \frac{1}{\varepsilon}\right)} \\
& =2^{\mathcal{O}\left(\frac{k^{4}}{\varepsilon^{2}} \log \frac{1}{\varepsilon}\right)} \cdot\left(\frac{1}{\varepsilon}\right)^{\mathcal{O}\left(\frac{k}{\varepsilon^{2}} \log \frac{1}{\varepsilon}\right)}
\end{aligned}
$$

Thus the running time of our algorithm is $2^{\mathcal{O}\left(\frac{k^{4}}{\varepsilon^{2}} \log \frac{1}{\varepsilon}\right)} \cdot\left(\frac{1}{\varepsilon}\right)^{\mathcal{O}\left(\frac{k}{\varepsilon^{2}} \log \frac{1}{\varepsilon}\right)} n \cdot m$.

Now we prove the correctness of the algorithm. Let $\widehat{k} \in[k]$ be the integer defined in Lemma 6. We know that $\operatorname{OPT}_{\widehat{k}}(J) \leq\left(1+\frac{\varepsilon}{4}\right) \operatorname{OPT}(J)$. This implies that there is $I \in\left(\begin{array}{c}{[k]} \\ \widehat{k}\end{array}\right)$ such that the $\mathrm{OPT}\left(J^{\prime}\right)$ is at most $\left(1+\frac{\varepsilon}{4}\right) \operatorname{OPT}(J)$, where $J^{\prime}=\left(X, \widehat{k}, \operatorname{proj}_{I}(\mathcal{R})\right)$. In the iteration of our algorithm corresponding to $I$, we get a solution $C_{I}^{\prime}$ to $J^{\prime}$ of cost at most $\left(1+\frac{\varepsilon}{160 k}\right)$ OPT $\left(J^{\prime}\right)$ with probability at least $1-\left(1-p\left(k^{*},|I|, \frac{\varepsilon}{4}\right)^{1 / p\left(k^{*},|I|, \frac{\varepsilon}{4}\right)} \geq 1-\frac{1}{e}\right.$. By Proposition $5, C_{I}$ is a solution to $J$ of cost at most $\left(1+\frac{\varepsilon}{160 k}\right)$ OPT $\left(J^{\prime}\right)$. Thus by Lemma 6 , the cost of $C_{I}$ is $\left(1+\frac{\varepsilon}{4}\right)\left(1+\frac{\varepsilon}{160 k}\right) \mathrm{OPT}(J) \leq(1+\varepsilon) \mathrm{OPT}(J)$. This completes the proof of the theorem.

\section{References}

[1] P. K. Agarwal, S. Har-Peled, and K. R. Varadarajan, Approximating extent measures of points, J. ACM, 51 (2004), pp. 606-635. 6

[2] N. Alon And B. Sudakov, On two segmentation problems, J. Algorithms, 33 (1999), pp. $173-184.6$

[3] N. Aмiт, The bicluster graph editing problem, Master's thesis, Tel Aviv University, 2004. 5

[4] S. Arora, R. Ge, R. Kannan, And A. Moitra, Computing a nonnegative matrix factorization - provably, in Proceedings of the 44th Annual ACM Symposium on Theory of Computing (STOC), ACM, 2012, pp. 145-162. 5

[5] M. Badoiu, S. Har-Peled, And P. Indyk, Approximate clustering via core-sets, in Proceedings of the 34th Annual ACM Symposium on Theory of Computing (STOC), ACM, 2002, pp. 250-257. 6 
[6] F. Ban, V. Bhattiprolu, K. Bringmann, P. Kolev, E. Lee, and D. P. Woodruff, A PTAS for $\ell \_p$-Low Rank Approximation, ArXiv e-prints, (2018). 6

[7] E. Bartl, R. Belohlávek, And J. Konecny, Optimal decompositions of matrices with grades into binary and graded matrices, Annals of Mathematics and Artificial Intelligence, 59 (2010), pp. 151-167. 6

[8] R. BelohláveK And V. Vychodil, Discovery of optimal factors in binary data via a novel method of matrix decomposition, J. Computer and System Sciences, 76 (2010), pp. 320. 6

[9] K. Bringmann, P. Kolev, And D. P. Woodruff, Approximation algorithms for $\ell_{0}$ low rank approximation, in Advances in Neural Information Processing Systems 30 (NIPS), 2017, pp. 6651-6662. 5

[10] L. S. Chandran, D. Issac, And A. Karrenbauer, On the parameterized complexity of biclique cover and partition, in Proceedings of the 11th International Symposium on Parameterized and Exact Computation (IPEC), vol. 63 of LIPIcs, Schloss Dagstuhl Leibniz-Zentrum fuer Informatik, 2016, pp. 11:1-11:13. 6

[11] K. L. Clarkson And D. P. WoodrufF, Input sparsity and hardness for robust subspace approximation, in Proceedings of the 56th Annual Symposium on Foundations of Computer Science (FOCS), IEEE Computer Society, 2015, pp. 310-329. 5

[12] C. Dan, K. A. Hansen, H. Jiang, L. Wang, And Y. Zhou, On low rank approximation of binary matrices, CoRR, abs/1511.01699 (2015). 5, 6

[13] F. V. Fomin, P. A. Golovach, And F. Panolan, Parameterized low-rank binary matrix approximation, CoRR, abs/1803.06102 (2018). 5

[14] _ Parameterized low-rank binary matrix approximation, CoRR, abs/1803.06102 (2018). 6

[15] Y. Fu, N. JiAng, AND H. Sun, Binary matrix factorization and consensus algorithms, in Proceedings of the International Conference on Electrical and Control Engineering (ICECE), IEEE, 2010, pp. 4563-4567. 5

[16] N. Gillis And S. A. Vavasis, On the complexity of robust PCA and $\ell_{1}$-norm low-rank matrix approximation, CoRR, abs/1509.09236 (2015). 5, 6

[17] J. Gramm, J. Guo, F. Hüffner, and R. Niedermeier, Data reduction and exact algorithms for clique cover, ACM Journal of Experimental Algorithmics, 13 (2008). 6

[18] D. A. Gregory, N. J. Pullman, K. F. Jones, And J. R. Lundgren, Biclique coverings of regular bigraphs and minimum semiring ranks of regular matrices, J. Combinatorial Theory Ser. B, 51 (1991), pp. 73-89. 6

[19] H. W. Gutch, P. Gruber, A. Yeredor, and F. J. Theis, ICA over finite fields separability and algorithms, Signal Processing, 92 (2012), pp. 1796-1808. 5

[20] W. Hoeffoing, Probability inequalities for sums of bounded random variables, Journal of the American Statistical Association, 58 (1963), pp. 13-30. 9

[21] P. Jiang and M. T. Heath, Mining discrete patterns via binary matrix factorization, in ICDM Workshops, IEEE Computer Society, 2013, pp. 1129-1136. 5 
[22] P. Jiang, J. Peng, M. Heath, And R. Yang, A clustering approach to constrained binary matrix factorization, in Data Mining and Knowledge Discovery for Big Data: Methodologies, Challenge and Opportunities, Springer Berlin Heidelberg, Berlin, Heidelberg, 2014, pp. 281-303. 5

[23] R. Kannan and S. Vempala, Spectral algorithms, Foundations and Trends in Theoretical Computer Science, 4 (2009), pp. 157-288. 5

[24] J. Kleinberg, C. Papadimitriou, and P. Raghavan, Segmentation problems, J. ACM, 51 (2004), pp. 263-280. 6

[25] M. Koyutürk and A. Grama, Proximus: A framework for analyzing very high dimensional discrete-attributed datasets, in Proceedings of the 9th ACM SIGKDD International Conference on Knowledge Discovery and Data Mining (KDD), New York, NY, USA, 2003, ACM, pp. 147-156. 5

[26] A. Kumar, Y. Sabharwal, and S. Sen, Linear-time approximation schemes for clustering problems in any dimensions, J. ACM, 57 (2010), pp. 5:1-5:32. 6, 7, 19

[27] H. Lu, J. Vaidya, V. Atluri, And Y. Hong, Constraint-aware role mining via extended boolean matrix decomposition, IEEE Trans. Dependable Sec. Comput., 9 (2012), pp. 655669. 6

[28] M. W. Mahoney, Randomized algorithms for matrices and data, Foundations and Trends in Machine Learning, 3 (2011), pp. 123-224. 5

[29] P. Miettinen, T. Mielikäinen, A. Gionis, G. Das, and H. Mannila, The discrete basis problem, IEEE Trans. Knowl. Data Eng., 20 (2008), pp. 1348-1362. 6

[30] P. Miettinen And J. VReEKen, Model order selection for boolean matrix factorization, in Proceedings of the 17th ACM SIGKDD International Conference on Knowledge Discovery and Data Mining (KDD), ACM, 2011, pp. 51-59. 6

[31] B. Mitra, S. Sural, J. Vaidya, and V. Atluri, A survey of role mining, ACM Comput. Surv., 48 (2016), pp. 50:1-50:37. 6

[32] M. Mitzenmacher and E. Upfal, Probability and Computing: Randomized Algorithms and Probabilistic Analysis, Cambridge University Press, New York, NY, USA, 2005. 9

[33] A. MoITRA, An almost optimal algorithm for computing nonnegative rank, SIAM J. Comput., 45 (2016), pp. 156-173. 5

[34] R. Ostrovsky and Y. Rabani, Polynomial-time approximation schemes for geometric min-sum median clustering, J. ACM, 49 (2002), pp. 139-156. 6

[35] A. Painsky, S. Rosset, And M. Feder, Generalized independent component analysis over finite alphabets, IEEE Trans. Information Theory, 62 (2016), pp. 1038-1053. 5

[36] I. P. Razenshteyn, Z. Song, And D. P. WoodrufF, Weighted low rank approximations with provable guarantees, in Proceedings of the 48th Annual ACM Symposium on Theory of Computing (STOC), ACM, 2016, pp. 250-263. 5

[37] B.-H. Shen, S. Ji, AND J. Ye, Mining discrete patterns via binary matrix factorization, in Proceedings of the 15th ACM SIGKDD International Conference on Knowledge Discovery and Data Mining (KDD), New York, NY, USA, 2009, ACM, pp. 757-766. 5 
[38] J. VAIDYA, Boolean matrix decomposition problem: Theory, variations and applications to data engineering, in Proceedings of the 28th IEEE International Conference on Data Engineering (ICDE), IEEE Computer Society, 2012, pp. 1222-1224. 6

[39] J. VAidya, V. AtLuRI, AND Q. Guo, The role mining problem: finding a minimal descriptive set of roles, in Proceedings of the 12th ACM Symposium on Access Control Models and (SACMAT), 2007, pp. 175-184. 6

[40] D. P. WoodrufF, Sketching as a tool for numerical linear algebra, Foundations and Trends in Theoretical Computer Science, 10 (2014), pp. 1-157. 5

[41] S. Wulff, R. URner, And S. Ben-David, Monochromatic bi-clustering, in Proceedings of the 30th International Conference on Machine Learning, (ICML), vol. 28 of JMLR Workshop and Conference Proceedings, JMLR.org, 2013, pp. 145-153. 5

[42] A. YEREDOR, Independent component analysis over Galois fields of prime order, IEEE Trans. Information Theory, 57 (2011), pp. 5342-5359. 5 